\title{
The Algebraic Index Theorem and Deformation Quantization of Lagrange-Finsler and Einstein Spaces
}

\author{
Sergiu I. Vacaru* \\ Rector's Office, Alexandru Ioan Cuza University, \\ Alexandru Lapuşneanu street, nr. 14, UAIC - Corpus R, office 323; \\ Iaşi, Romania, 700057
}

July 1, 2013

\begin{abstract}
Various types of Lagrange and Finsler geometries, Einstein gravity, and modifications, can be modelled by nonholonomic distributions on tangent bundles/ manifolds when the fundamental geometric objects are adapted to nonlinear connection structures. We can convert such geometries and physical theories into almost Kähler/ Poisson structures on (co)tangent bundles. This allows us to apply deformation quantization formalism to almost symplectic connections induced by Lagrange-Finsler and/or Einstein fundamental geometric objects. There are constructed respective nonholonomic versions of the trace density maps for the zeroth Hochschild homology of deformation quantization of distinguished algebras (in this work, adapted to nonlinear connection structure). Our main result consists in an algebraic index theorem for Lagrange-Finsler and Einstein spaces. Finally, we show how the Einstein field equations for gravity theories and geometric mechanics models can be imbedded into the formalism of deformation quantization and index theorem.
\end{abstract}

Keywords: Deformation quantization, algebraic index theorems, Lagrange-Finsler geometry, nonholonomic Einstein manifolds, quantum gravity.

MSC2000: 16E40, 19K56, 46L65, 46M20, 53B35, 53B40, 53C15, 53D $55,58 \mathrm{~J} 22,81 \mathrm{~S} 10,83 \mathrm{C} 45,83 \mathrm{C} 99$

*sergiu.vacaru@uaic.ro 


\section{Introduction}

The goal of this paper is to prove an algebraic index theorem for generalized Finsler and/or Einstein spaces and show how corresponding gravitational field equations, and their solutions, can be encoded into a nonholonomic version of Fedosov manifolds for deformation quantization. It is a partner work of [30, [5] and belongs to a series of our articles on geometric methods in deformation, A-brane, bi-connection etc quantization models of gravity and gauge theories and geometric mechanics [28, 29, 32, 33, 13. The main motivations for this kind of investigations come from classical and quantum nonlinear fundamental physical equations encoded as geometric structures on nonholonomic manifolds [1], and there is an important physical task to quantize such generic nonlinear theories. Similar constructions arise also as mathematical problems for developing quantum/ noncommutative versions of Riemann-Finsler and Hamilton-Lagrange spaces and in relation to possible applications of geometric methods in modern particle physics.

The concept of nonholonomic manifold came from geometric mechanics and classical and quantum theories with non-integrable (equivalently, nonholonomic, or anholonomic) constraints [2]. In our works, we follow certain methods and formalism developed in the geometry of classical and quantum nonholonomic manifolds, Lagrange - Finsler/ Hamilton - Cartan geometries and applications [38, 39, 40], see comprehensive reviews and references in [7, 31]. It allows us to elaborate an unified geometric approach for the above mentioned types of classical and quantum models, working, for simplicity, with nonholonomic distributions defining nonlinear connection (N-connection) structures via non-integrable splitting into Whitney sums of corresponding tangent spaces (see next section for a summary of necessary definitions and results).

It is well-known that the algebra of pseudo-differential operators on a compact manifold $M$ can be viewed as a quantum model (i.e. quantization) of the cotangent bundle $T^{*} M$. In this framework, the Atiyah-Singer index theorem [6] relates the index of an elliptic pseudo-differential operator on $M$ to the Todd class of $M$, when the Chern character of the bundle is associated naturally with the symbol of the pseudo-differential operator under consideration. B. Fedosov developed a deformation quantization (via formal power series with complex coefficients) of an arbitrary symplectic manifold $\mathcal{M}$ [14, 15]. A natural analogue of index theorem was proposed in $\mathrm{K}$-theory working with the quantum algebra of functions on symplectic manifolds. Here we note that quantum deformations use the correspondence between physical structures and deformations of algebraic noncommutative structures.

However, the bulk of physical theories usually are not encoded in terms of the geometry of "pure" symplectic manifolds or further developments as 
Poisson manifolds and their deformation quantization [23, 24]. A quite universal geometric scheme including (semi) Riemannian [3] and Finsler spaces, Lagrange and Hamilton mechanics and generalized nonholonomic Einstein spaces can be elaborated in the framework of the geometry of almost Kähler manifolds [30, 31, 32] with associated canonical N-connection structure. This scheme can be generalized for additional geometric/physical structures which implies new important results in geometric and/or deformation quantization. For instance, we cite some applications of Fedosov's quantization in modern particle physics (see, for instance, [11, 18]) and for almost symplectic geometry, in general, with nontrivial torsion, see [21, 20, 19].

In order to elaborate explicit models of deformation quantization for generally constrained physical systems, the most important task was to prove that the fundamental geometric objects defining such almost symplectic spaces are determined naturally, and in a unique form, by generating (fundamental) Finsler/Lagrange functions, generic off-diagonal metrics( in particular, being solutions of the Einstein equations and/or generalizations). Such a project was derived from Fedosov works [14, 15] and KarabegovSchlichenmaier developments [21] for deformation quantization of almost Kähler geometries and realized in a series of our works [28, 29, 30, 5].

Generalizations of the famous Atiyah-Singer index theorem for nonholonomic Clifford bundles, in particular, generated for Lagrange-Finsler spaces, and gerbes were studied in Refs. [36]. Nevertheless, those versions do not provide straightforward relations to almost Kähler models of Finsler/-Lagrange and/or Cartan/-Hamilton geometries, Einstein manifolds and their deformation quantization. In this article, we provide a local version and a simple proof of the algebraic index theorem for the mentioned types of nonholonomic almost symplectic manifolds. We follow the scheme using an explicit formula for the trace density map from the quantum algebra of functions on an arbitrary symplectic manifold $\mathcal{M}$ to the top degree cohomology of $\mathcal{M}$ [16]. In our case, that formalism is adapted to $\mathrm{N}$-connection structures. More precisely, our constructions are built from fundamental geometric objects derived from canonical almost symplectic forms and nonlinear and linear connections for almost Kähler-Finsler manifolds (and various modifications for Lagrange-Hamilton geometric mechanics and/or the Einstein gravity theory).

Throughout the paper we assume the summation over repeated right indices and use left indices as abstract labels for certain geometric objects; boldface symbols being considered for spaces/objects which are enabled/adapted to nonlinear connection structures (such a system of notations was elaborated in Ref. [31]).

The paper is organized as follows. In section 2, we summarize the necessary results on almost Kähler geometric models of Lagrange-Finsler and Einstein spaces. Section 3 is devoted to Fedosov quantization of EinsteinFinsler spaces and the formalism of trace density maps adapted to nonlinear 
connection structure. We prove the Main Result (a local Atiyah-Singer index theorem for Lagrange-Finsler and Einstein Spaces) in section 4. The problem of algebraic index encoding of Einstein equations and exact solutions in gravity theories is also discussed.

Acknowledgement: The work is partially supported by the Program IDEI, PN-II-ID-PCE-2011-3-0256. The author is grateful to organizers and participants of "Applied Seminar" at Department of Mathematics, University College of London, UK; organizers of the Conference 3 Quantum: Algebra, Geometry, Information (Tallinn University of Technology, Estonia; July 10-14, 2012) and XXXI Workshop on Geometric Methods in Physics, Bialowieza, Poland, June 24-30, 2012, where the results of this paper where presented in memory of Prof. B. Fedosov. The author thanks the Referee for important requests and suggestions which improved substantially the content of the article.

\section{Almost Kähler Models of Lagrange-Finsler \& Einstein Spaces}

In this section, we recall some necessary results on encoding data for nonholonomic manifolds/bundles as almost Kähler spaces with fundamental geometric objects adapted to nonlinear connection (N-connection) structure.

\subsection{Nonholonomic distributions with associated $\mathrm{N}$-connecti- on}

Let us consider a $(n+m)$-dimensional nonholonomic manifold (see definition in note [?]) $\mathbf{V}, \operatorname{dim} \mathbf{V} \geq 2+1$, of necessary smooth class. Such a space is enabled with a conventional $n+m$ splitting when local coordinates $u=(x, y)$ on an open region $U \subset \mathbf{V}$ are labeled in the form $u^{\alpha}=\left(x^{i}, y^{a}\right)$, $\alpha=(i, a)$, where indices $i, j, k, \ldots=1,2, \ldots, n$ and $a, b, c \ldots=n+1, \ldots, n+m$. There are changes of coordinates $\left(x^{i}, y^{a}\right) \rightarrow\left(\tilde{x}^{i}, \tilde{y}^{a}\right)$ when $\tilde{x}^{i}$ are functions only of $x^{i}$. It results that $\frac{\partial}{\partial y^{a}}=\frac{\partial \tilde{y}^{b}}{\partial y^{a}} \frac{\partial}{\partial y^{a}}$ and so $\frac{\partial}{\partial y^{a}}$ locally span an integrable distribution on $\mathbf{V}$. We may fix a supplement of it locally spanned by $\mathbf{e}_{i}=\delta_{i}=\frac{\partial}{\partial x^{i}}-N_{i}^{a}(u) \frac{\partial}{\partial y^{a}}$. This is an example of non-integrable distribution $\mathcal{N}$ transforming $\mathbf{V}$ into a nonholonomic manifold. If the functions $N_{i}^{a}(u)$ are chosen in such a way that $\delta_{j}=\frac{\partial \tilde{x}^{i}}{\partial x^{j}} \delta_{i}$, one says that $\mathbf{N}=\left\{N_{i}^{a}(u)\right\}$ defines a nonlinear connection structure. In particular, $\mathbf{V}$ can be the total space of submersion over a $n$-dimensional manifold $M$. If $\mathbf{V}=T M$, where $T M$ is the total space of a tangent bundle to a $n$-dimensional manifold $M$, the $\mathrm{N}$-connection structure can be considered any one introduced for a (pseudo) Finsler, or Lagrange, geometry modeled on such a tangent bundle [31]. For $v T M$ being the vertical distributions on $T M$, we introduce: 
Definition 2.1 Any Whitney sum

$$
T T M=h T M \oplus v T M
$$

defines a nonlinear connection ( $N$-connection) structure parametrized by local vector fields $e_{i}=\frac{\partial}{\partial x^{i}}-N_{i}^{a}(x, y) \frac{\partial}{\partial y^{a}}$ on $T M$.

A N-connection states on $T M$ a conventional horizontal (h) and vertical (v) splitting (decomposition). If a $\mathrm{h}-\mathrm{v}$ splitting, $T \mathbf{V}=h \mathbf{V} \oplus v \mathbf{V}$, exists on a general nonholonomic manifold $\mathbf{V}$, we call such a space $\mathrm{N}$-anholonomic. For gravity theories, we shall consider $\mathbf{V}$ to be a (pseudo) Riemannian spacetime.

Let $L(x, y)$ be a regular differentiable Lagrangian on $U \subset T M$ (for analogous models $U \subset \mathbf{V}$ ) with non-degenerate Hessian (equivalently, fundamental tensor field)

$$
g_{a b}(x, y)=\frac{\partial^{2} L}{\partial y^{a} \partial y^{b}} .
$$

Definition 2.2 ([22, 31]) A Lagrange space $L^{n}=(M, L(x, y))$ is defined by a function $T M \ni(x, y) \rightarrow L(x, y) \in \mathbb{R}$, i.e. a fundamental Lagrange function, which is differentiable on $\widehat{T M}:=T M \backslash\{0\}$, where $\{0\}$ is the set of null sections, and continuous on the null section of $\pi: T M \rightarrow M$ and such that the (Hessian) tensor field $g_{a b}(x, y)$, (2), is non-degenerate and of constant signature on $\widetilde{T M}$.

A (pseudo) Lagrange space can be effectively modelled on a nonholonomic (pseudo) Riemann manifold of even dimension $\mathbf{V}=\left(\mathbf{V}^{2 n}, \underline{\mathbf{g}}\right), \operatorname{dim} \mathbf{V}^{2 n}=$ $2 n, n \geq 2$, prescribing a generating function $\mathcal{L}(x, y)$, with $u=(x, y) \in \mathbf{V}^{2 n}$, satisfying the conditions of Definition 2.2. We shall use two symbols $L$ and $\mathcal{L}$ in order to distinguish what type of geometric mechanical model (a classical one, from Lagrange geometry, or an analogous, pseudo-Lagrange one, on a pseudo-Riemannian manifold) we involve in our constructions.

Let us consider a regular curve $u(\tau)$ with real parameter $\tau$, when $u: \tau \in$ $[0,1] \rightarrow x^{i}(\tau) \subset U$. It can be lifted to $\pi^{-1}(U) \subset \widetilde{T M}$ as $\widetilde{u}(\tau): \tau \in[0,1] \rightarrow$ $\left(x^{i}(\tau), y^{i}(\tau)=\frac{d x^{i}}{d \tau}\right)$ since the vector field $\frac{d x^{i}}{d \tau}$ does not vanish on $\widetilde{T M}$. For a different calculus [31], we prove:

Theorem 2.1 The Euler-Lagrange equations, $\frac{d}{d \tau} \frac{\partial L}{\partial y^{i}}-\frac{\partial L}{\partial x^{i}}=0$, are equivalent to the nonlinear geodesic (semi-spray) equations

$$
\frac{d^{2} x^{i}}{d \tau^{2}}+2 G^{i}(x, y)=0
$$

where $G^{i}=\frac{1}{2} g^{i j}\left(\frac{\partial^{2} L}{\partial y^{j} \partial x^{k}} y^{k}-\frac{\partial L}{\partial x^{j}}\right)$, for $g^{i j}$ being the inverse to $g_{i j}$ (2). 
The conditions, and proof, of this theorem can be redefined on arbitrary $\left(\mathbf{V}^{2 n}, \underline{\mathbf{g}}\right)$ endowed with a nonholonomic distribution induced by any prescribed $\mathcal{L}$ subjected to conditions similar to those for a regular Lagrangian in mechanics.

Proposition 2.1 There are canonical frame and co-frame structures, $\mathbf{e}_{\alpha}$ and $\mathbf{e}^{\alpha}$, respectively defined by the canonical $N$-connection

$$
{ }^{c} N_{i}^{a}:=\frac{\partial G^{a}}{\partial y^{i}}
$$

when [4]

$$
\begin{aligned}
\mathbf{e}_{\alpha} & =\left(\mathbf{e}_{i}=\frac{\partial}{\partial x^{i}}-N_{i}^{a} \frac{\partial}{\partial y^{a}}, e_{b}=\frac{\partial}{\partial y^{b}}\right) \\
\mathbf{e}^{\alpha} & =\left(e^{i}=d x^{i}, \mathbf{e}^{b}=d y^{b}+N_{i}^{b} d x^{i}\right)
\end{aligned}
$$

for $\left.\mathbf{e}_{\alpha}\right\rfloor \mathbf{e}^{\beta}=\delta_{\alpha}^{\beta}$, where by $\rfloor$ we note the interior products and $\delta_{\alpha}^{\beta}$ being the Kronecker delta symbol. Such $N$-elongated partial derivative/ differential operators can be defined for any sets $N_{i}^{a}$ which are not obligatory represented in a canonical form ${ }^{c} N_{i}^{a}$ ).

Proof. The results can be proven on any $\mathrm{N}$-anholnomic manifold $\mathbf{V}$ by explicit constructions using formula (4) with $G^{a}$ determined in (3); it is imposed the condition that such (co) frames should depend linearly on coefficients of respective $\mathrm{N}$-connections.

Definition 2.3 The $N$-lift of the fundamental tensor fields $g_{a b}$ (2) on TM (in general, from any $h \mathbf{V}$ to $\mathbf{V}$ ) is a Sasaki type metric (distinguished metric, d-metric)

$$
\mathbf{g}=\mathbf{g}_{\alpha \beta} \mathbf{e}^{\alpha} \otimes \mathbf{e}^{\beta}=g_{i j}(x, y) e^{i} \otimes e^{j}+g_{a b}(x, y) \mathbf{e}^{a} \otimes \mathbf{e}^{b},
$$

where $g_{i j}$ is stated by $g_{a b}$ following $g_{i j}=g_{n+i n+j}$.

Canonical N-connection and d-metric structures can be constructed on any (pseudo) Riemannian manifold of even dimension, $\mathbf{V}=\left(\mathbf{V}^{2 n}, \underline{\mathbf{g}}\right)$, dim $\mathbf{V}^{2 n}=2 n, n \geq 2$, with given metric structure $\underline{\mathbf{g}}=\mathbf{g}_{\underline{\alpha} \beta} \partial^{\underline{\alpha}} \otimes \partial^{\underline{\beta}}$ if a generating Lagrange function $\mathcal{L}(x, y)$ is correspondingly prescribed on $\mathbf{V}^{2 n}$. Any metric g can be represented in a Lagrange-Sasaki form (7) via frame transforms

$$
\mathbf{e}_{\alpha}=\mathbf{e}_{\alpha}^{\underline{\alpha}} \partial_{\underline{\alpha}} \text { and } \mathbf{e}^{\alpha}=\mathbf{e}_{\underline{\alpha}}^{\alpha} \partial^{\underline{\alpha}} ; \mathbf{g}_{\alpha \beta}=\mathbf{e}_{\alpha}^{\underline{\alpha}} \mathbf{e}_{\beta}^{\underline{\beta}} \mathbf{g}_{\underline{\alpha} \underline{\beta}},
$$

where matrices $\mathbf{e}_{\alpha}^{\underline{\alpha}}$ and $\mathbf{e}_{\underline{\alpha}}^{\alpha}$ can be chosen to be mutually inverse. The explicit formulas depend on the type of nonholonomic structure we prescribe by $\mathcal{L}(x, y)$ (via induced $\mathrm{N}$-connection (4) and $\mathrm{N}$-adapted frames (5) and 
(6) ). For instance, if $\mathbf{g}_{\underline{\alpha} \beta}$ is given as a solution of the Einstein equations in four dimensional (4-d) general relativity and $\mathbf{g}_{\alpha \beta}$ is determined by a chosen $\mathcal{L}$, we can always define certain $\mathbf{e}_{\alpha}^{\underline{\alpha}}$ encoding the gravitational data into analogous mechanical ones, and/or inversely. In 4-d gravity, there are six independent components of $\mathbf{g}_{\underline{\alpha} \beta}$ (from ten ones for a symmetric second rank tensor, we can always fix four ones by corresponding coordinate transforms). The values $\mathbf{e}_{\alpha}^{\underline{\alpha}}$ can be defined as some solutions of algebraic equations (8) for given coefficients of metrics. Such constructions allow us always to introduce (pseudo) Lagrange variables on a (pseudo) Riemannian manifold and, inversely, any regular Lagrange mechanics can be geometrized as a Riemannian space enabled with additional nonholonomic structure determined by $L(x, y)$.

We have the following:

Proposition 2.2 A canonical $N$-connection $\mathbf{N}$ (4) defines a canonical almost complex structure $\mathbf{J}$.

Proof. The linear operator $\mathbf{J}$ acting on $\mathbf{e}_{\alpha}=\left(\mathbf{e}_{i}, e_{b}\right)$ (5) is defined by

$$
\mathbf{J}\left(\mathbf{e}_{i}\right)=-\mathbf{e}_{n+i} \text { and } \mathbf{J}\left(e_{n+i}\right)=\mathbf{e}_{i} .
$$

This is a global almost complex structure $(\mathbf{J} \circ \mathbf{J}=-\mathbf{I}$ for $\mathbf{I}$ being the unity matrix) on $T M$ completely determined by $L(x, y)$.

Definition 2.4 The Neijenhuis tensor field for an almost complex structure $\mathbf{J}$ determined by a $\mathrm{N}$-connection (i.e. the curvature of $N$-connection) is

$$
{ }^{\mathbf{J}} \boldsymbol{\Omega}(\mathbf{X}, \mathbf{Y}):=-[\mathbf{X}, \mathbf{Y}]+[\mathbf{J X}, \mathbf{J Y}]-\mathbf{J}[\mathbf{J X}, \mathbf{Y}]-\mathbf{J}[\mathbf{X}, \mathbf{J Y}],
$$

for any vectors $\mathbf{X}$ and $\mathbf{Y}$.

The N-adapted (co) bases (5) and (6) are nonholonomic when $\left[\mathbf{e}_{\alpha}, \mathbf{e}_{\beta}\right]=$ $\mathbf{e}_{\alpha} \mathbf{e}_{\beta}-\mathbf{e}_{\beta} \mathbf{e}_{\alpha}=W_{\alpha \beta}^{\gamma} \mathbf{e}_{\gamma}$. The nontrivial (antisymmetric) anholonomy coefficients are $W_{i a}^{b}=\partial_{a} N_{i}^{b}$ and $W_{j i}^{a}=\Omega_{i j}^{a}$, with the coefficients of N-connection curvature computed $\Omega_{i j}^{a}=\frac{\partial N_{i}^{a}}{\partial x^{j}}-\frac{\partial N_{j}^{a}}{\partial x^{i}}+N_{i}^{b} \frac{\partial N_{j}^{a}}{\partial p_{b}}-N_{j}^{b} \frac{\partial N_{i}^{a}}{\partial p_{b}}$.

We can introduce on a (pseudo) Riemannian manifold an analogous (pseudo) Finsler structure defined for any $\mathcal{L}=\mathcal{F}^{2}(x, y)$, where an effective Finsler metric $\mathcal{F}$ is a differentiable function of class $C^{\infty}$ in any point $(x, y)$ with $y \neq 0$ and is continuous in any point $(x, 0) ; \mathcal{F}(x, y)>0$ if $y \neq 0$; it satisfies the homogeneity condition $\mathcal{F}(x, \beta y)=|\beta| \mathcal{F}(x, y)$ for any nonzero $\beta \in \mathbb{R}$ and the Hessian (2) computed for $\mathcal{L}=\mathcal{F}^{2}$ is positive definite. A nonholonomic manifold can be alternatively modelled equivalently as an analogous Finsler space. On convenience, in this work, we shall consider both types of alternative modelling of gravity theories (with nonhomogeneous configurations, i.e. Lagrange type, and homogeneous ones, i.e. Finsler type). 


\section{$2.2 \quad \mathrm{~N}$-adapted almost Kähler structures}

An almost Kähler geometry can be also adapted to (induced by) canonical $\mathrm{N}$-connections.

Definition 2.5 An almost symplectic structure on $\mathbf{V}$ is defined by a nondegenerate 2-form $\theta=\frac{1}{2} \theta_{\alpha \beta}(u) e^{\alpha} \wedge e^{\beta}$.

An almost Hermitian model of a nonholonomic (pseudo) Riemannian space $\mathbf{V}^{2 n}, \operatorname{dim} \mathbf{V}^{2 n}=2 n, n \geq 1$, equipped with an $\mathrm{N}$-connection structure $\mathbf{N}$ is defined by a triple $\mathbf{H}^{2 n}=\left(\mathbf{V}^{2 n}, \theta, \mathbf{J}\right)$, where $\theta(\mathbf{X}, \mathbf{Y}) \doteqdot \mathbf{g}(\mathbf{J X}, \mathbf{Y})$. In addition, we have that a space $\mathbf{H}^{2 n}$ is almost Kähler, denoted $\mathbf{K}^{2 n}$, if and only if $d \theta=0$. In this paper, we consider that a real manifold is almost Kähler if it is endowed with a closed almost symplectic 2-form $\theta$.

We recall that for pseudo-Lagrange/ Finsler modelling of Einstein gravity [29, 30, 31] (see also discussion and references therein; for Finsler spaces, the original result is due to [25]):

Theorem 2.2 Having chosen a generating function $\mathcal{L}(x, y)$ (or $\mathcal{F}(x, y))$ on a (pseudo) Riemannian manifold $\mathbf{V}^{2 n}$, we can model this space as an almost Kähler geometry, i.e. ${ }^{\mathcal{L}} \mathbf{H}^{2 n}={ }^{\mathcal{L}} \mathbf{K}^{2 n}$, where the left labels emphasize that such structures are induced nonholonomically by $\mathcal{L}($ or $\mathcal{F})$.

Let us consider a metric $\mathbf{g}(7)$ and some structures $\mathbf{N}$ and $\mathbf{J}$ canonically defined by a prescribed $\mathcal{L}$. We define $\theta(\mathbf{X}, \mathbf{Y}) \doteqdot \mathbf{g}(\mathbf{J X}, \mathbf{Y})$ for any vectors $\mathbf{X}$ and $\mathbf{Y}$ and compute locally

$$
\begin{aligned}
\theta & =\frac{1}{2} \theta_{\alpha \beta}(u) e^{\alpha} \wedge e^{\beta}=\frac{1}{2} \theta_{\underline{\alpha} \underline{\beta}}(u) d u^{\underline{\alpha}} \wedge d u^{\underline{\beta}} \\
& =g_{i j}(x, y) e^{n+i} \wedge d x^{j}=g_{i j}(x, y)\left(d y^{n+i}+N_{k}^{n+i} d x^{k}\right) \wedge d x^{j}
\end{aligned}
$$

Introducing the the form $\omega=\frac{1}{2} \frac{\partial \mathcal{L}}{\partial y^{n+i}} d x^{i}$, we get $\theta=d \omega$, i.e. $d \theta=d d \omega=0$. We conclude that using a generating function $\mathcal{L}$ (or $\mathcal{F}$ ), via canonical $\mathbf{g}, \mathbf{N}$ and $\mathbf{J}$, a (pseudo) Riemannian/Finsler/Lagrange space can be represented equivalently as an almost Kähler geometry.

Definition 2.6 A linear connection on $\mathbf{V}^{2 n}$ is a distinguished connection (d-connection) $\mathbf{D}=(h D ; v D)=\left\{\boldsymbol{\Gamma}_{\beta \gamma}^{\alpha}=\left(L_{j k}^{i},{ }^{v} L_{b k}^{a} ; C_{j c}^{i},{ }^{v} C_{b c}^{a}\right)\right\}$, with local coefficients computed with respect to (5) and (6), which preserves the distribution (1) under parallel transports.

A d-connection $\mathbf{D}$ is metric compatible with a d-metric $\mathbf{g}$ if $\mathbf{D}_{\mathbf{X}} \mathbf{g}=0$ for any $\mathrm{d}-$-vector field $\mathbf{X}$.

Definition 2.7 An almost symplectic d-connection ${ }_{\theta} \mathbf{D}$ on $\mathbf{V}^{2 n}$ (equivalently, we can say that a $d$-connection is compatible with an almost symplectic structure $\theta$ ) is defined such that ${ }_{\theta} \mathbf{D}$ is $N$-adapted, i.e., it is a d-connection, and ${ }_{\theta} \mathbf{D}_{\mathbf{X}} \theta=0$, for any $d$-vector $\mathbf{X}$. 
For N-anholonomic manifolds of even dimension, we have the following:

Theorem 2.3 There is a unique normal d-connection

$$
\begin{aligned}
\widehat{\mathbf{D}} & =\left\{h \widehat{D}=\left(\widehat{D}_{k},{ }^{v} \widehat{D}_{k}=\widehat{D}_{k}\right) ; v \widehat{D}=\left(\widehat{D}_{c},{ }^{v} \widehat{D}_{c}=\widehat{D}_{c}\right)\right\} \\
& =\left\{\widehat{\boldsymbol{\Gamma}}_{\beta \gamma}^{\alpha}=\left(\widehat{L}_{j k}^{i},{ }^{v} \widehat{L}_{n+j n+k}^{n+i}=\widehat{L}_{j k}^{i} ; \widehat{C}_{j c}^{i}={ }^{v} \widehat{C}_{n+j c}^{n+i},{ }^{v} \widehat{C}_{b c}^{a}=\widehat{C}_{b c}^{a}\right)\right\},
\end{aligned}
$$

which is metric compatible, $\widehat{D}_{k} g_{i j}=0$ and $\widehat{D}_{c} g_{i j}=0$, and completely defined by $\mathbf{g}$ and a prescribed $\mathcal{L}(x, y)$.

Proof. Choosing

$$
\widehat{L}_{j k}^{i}=\frac{1}{2} g^{i h}\left(\mathbf{e}_{k} g_{j h}+\mathbf{e}_{j} g_{h k}-\mathbf{e}_{h} g_{j k}\right), \widehat{C}_{j k}^{i}=\frac{1}{2} g^{i h}\left(\frac{\partial g_{j h}}{\partial y^{k}}+\frac{\partial g_{h k}}{\partial y^{j}}-\frac{\partial g_{j k}}{\partial y^{h}}\right),
$$

we construct such a d-connection $\widehat{\mathbf{D}}_{\alpha}=\left(\widehat{D}_{k}, \widehat{D}_{c}\right)$, with $\mathrm{N}$-adapted coefficients $\widehat{\boldsymbol{\Gamma}}_{\beta \gamma}^{\alpha}=\left(\widehat{L}_{j k}^{i},{ }^{v} \widehat{C}_{b c}^{a}\right)$.

We provide the $\mathrm{N}$-adapted formulas for torsion and curvature of the normal d-connection in Appendix A.

For the purposes of this work, this property of the normal d-connection is very important (it follows from a straightforward verification):

Theorem 2.4 The normal $d$-connection $\widehat{\mathbf{D}}$ defines a unique almost symplectic d-connection, $\widehat{\mathbf{D}} \equiv{ }_{\theta} \widehat{\mathbf{D}}$, which is $N$-adapted, i.e. it preserves under parallelism the splitting (1), ${ }_{\theta} \widehat{\mathbf{D}}_{\mathbf{X}} \theta=0$ and $\widehat{T}_{j k}^{i}=\widehat{T}_{b c}^{a}=0$, see (A.5).

We note that the normal d-connection $\widehat{\boldsymbol{\Gamma}}_{\beta \gamma}^{\alpha}$ is a N-anholonomic analog of the affine connection ${ }^{K} \boldsymbol{\Gamma}_{\beta \gamma}^{\alpha}$ and Nijenhuis tensor ${ }^{K} \boldsymbol{\Omega}_{\beta \gamma}^{\alpha}$ with the torsion satisfying the condition ${ }^{K} \mathbf{T}_{\beta \gamma}^{\alpha}=(1 / 4)^{K} \boldsymbol{\Omega}^{\alpha}{ }_{\beta \gamma}$ considered in Ref. [21] (those constructions were not for spaces enabled with $\mathrm{N}$-connection structure). On $\mathrm{N}$-anholonomic manifolds, we can work equivalently with both types of linear connections.

\section{Nonholonomic Fedosov Quantization}

We modify the Fedosov method in a form which allows us to quantize the Lagrange-Finsler and Einstein spaces and related generalizations for Einstein-Finsler quantum gravity models. Nonholonomic Chern-Weil homomorphisms are defined. Our former results on nonholonomic deformation quantization from [28, 29, 30] are revised with the aim to elaborate in the next section a $\mathrm{N}$-adapted trace density map formalism (originally considered in [16, 12]) and prove the local index theorem for $\mathrm{N}$-anholonomic manifolds. 


\subsection{Nonholonomic Chern-Weil homomorphisms}

Let us consider a complex vector space $\mathcal{V}^{2 n}$, $\operatorname{dim} \mathcal{V}^{2 n}=2 n$, endowed with a symplectic form $b^{\alpha \beta}=-b^{\beta \alpha}$; coefficients are defined with respect to a local coordinate basis $e_{\alpha}=\partial_{\alpha}=\partial / \partial u^{\alpha}$, for $\alpha, \beta, \ldots=1,2, \ldots, 2 n$. We call ${ }^{d} \mathcal{V}=(h \mathcal{V}, v \mathcal{V})=\mathcal{V}^{2 n}$ to be a distinguished vector (d-vector) space if it is enabled with a conventional $\mathrm{h}-$ and $\mathrm{v}$-splitting of type (1) into two symplectic subspaces with $b^{\alpha \beta}=\left(b^{i j}=-b^{j i}, b^{a c}=-b^{c a}\right)$ and $z^{\alpha}=\left(z^{i}, z^{a}\right)$. On $\mathrm{N}$-anholonomic manifolds, there are considered (distinguished) d-vector, d-tensor, d-connection, d-spinor, d-group, d-algebra etc fields which can be adapted to $\mathrm{N}$-connection structure into certain "irreducible" h-v-components.

Deformation quantization is elaborated for formal extensions on a "small" parameter $v$. In various papers, authors prefer to write $v=\hbar$ considering certain analogy with the Plank constant. We associate to a symplectic dvector space ${ }^{d} \mathcal{V}$ :

Definition 3.1 The Weyl d-algebra ${ }^{d} \mathcal{W}=(h \mathcal{W}, v \mathcal{W})$ over the field $\mathbb{C}((v))$ is the $d$-vector space $\mathbb{C}\left[\left[{ }^{d} \mathcal{V}\right]\right](v)$ of the formally completed symmetric $d^{-}$ algebra of ${ }^{d} \mathcal{V}$ endowed with associative multiplication (Wick product)

$$
\begin{aligned}
{ }^{1} q \circ{ }^{2} q(z, v):= & \exp \left(i \frac{v}{4} b^{\alpha \beta}\left(\frac{\delta^{2}}{\partial z^{\alpha} \partial{ }^{1} z^{\beta}}+\frac{\delta^{2}}{\partial{ }^{1} z^{\beta} \partial z^{\alpha}}\right)\right) \times \\
& \left.{ }^{1} q(z, v){ }^{2} q\left({ }^{1} z, v\right)\right|_{z=1}{ }^{z} \\
= & \exp \left(i \frac{v}{4} b^{i j}\left(\frac{\delta^{2}}{\partial z^{i} \partial{ }^{1} z^{j}}+\frac{\delta^{2}}{\partial{ }^{1} z^{j} \partial z^{i}}\right)+i \frac{v}{2}\left(b^{a c} \frac{\delta^{2}}{\partial z^{a} \partial{ }^{1} z^{c}}\right)\right) \\
& \times\left.{ }^{1} q(z, v)^{2} q\left({ }^{1} z, v\right)\right|_{z=}{ }^{1} z,
\end{aligned}
$$

for any ${ }^{1} q,{ }^{2} q \in{ }^{d} \mathcal{W}$.

In $\mathrm{h}-\mathrm{v}$-components the product (11), we use $\mathrm{N}$-elongated derivatives $\delta / \partial z^{\alpha}$ and $\delta / \partial{ }^{1} z^{\alpha}$ of type (5) considering that, in general, we work with nonolonomic distributions on vector spaces, algebras and manifolds/ bundles. All constructions can be redefined for usual partial derivatives with respect to local coordinate frames. There are natural $\mathrm{h}-$ and $\mathrm{v}-$ filtrations, correspondingly, of $h \mathcal{W}$ and $v \mathcal{W}$ constructed with respect to the degrees of monomials $2[v]+\left[z^{i}\right]$ and $2 v+\left[z^{a}\right]$. These filtrations define corresponding "N-adapted" $\left(2[v]+\left[z^{i}\right]\right)$-adic and $\left(2[v]+\left[z^{a}\right]\right)$-adic topologies parametrized in the form

$$
\begin{aligned}
\ldots \quad \subset \quad h \mathcal{W}^{1} \subset h \mathcal{W}^{0} \subset h \mathcal{W}^{-1} \ldots \subset h \mathcal{W}, \\
\text { for } h \mathcal{W}^{h_{s}}=\left\{\sum_{2 k+p \geq h_{s}} v^{k}{ }^{k} a_{i_{i} \ldots i_{p}}\left(u^{\alpha}\right) z^{i_{1}} \ldots z^{i_{p}}\right\} ; \\
\ldots \quad \subset \quad v \mathcal{W}^{1} \subset v \mathcal{W}^{0} \subset v \mathcal{W}^{-1} \ldots \subset v \mathcal{W}, \\
\text { for } v \mathcal{W}^{v^{s}}=\left\{\sum_{2 k+p \geq{ }^{\prime} v_{s}} v^{k} a_{b_{i} \ldots b_{p}}\left(u^{\alpha}\right) z^{b_{1}} \ldots z^{b_{p}}\right\},
\end{aligned}
$$


for $i_{1}, i_{2}, \ldots i_{p}=1,2, \ldots, n$ and $b_{1}, b_{2}, \ldots, b_{p}=n+1, \ldots n+n$.

For Weyl d-algebras, we have to adapt to $\mathrm{N}$-connections the explicit constructions with the $(n+n)$-th Hochschild cocycle $C^{n+n}\left({ }^{d} \mathcal{W},{ }^{d} \mathcal{W}^{*}\right)$ with coefficients in the dual $\mathrm{d}$-module of ${ }^{d} \mathcal{W}^{*}=\left(h \mathcal{W}^{*}, v \mathcal{W}^{*}\right)$. Let us consider a $(n+n)$-th simplex (with standard orientation) $\triangle_{2 n}=\left\{\left(p_{1}, \ldots, p_{2 n}\right) \in\right.$ $\left.\mathbb{R}^{2 n} ; p_{1} \leq \ldots \leq p_{2 n} \leq 1\right\}$ and denote the natural projection from ${ }^{d} \mathcal{W}^{\otimes(n+n+1)}$ onto $\mathbb{C}((v))$ by $\mu_{2 n}\left(q_{0} \otimes \ldots \otimes q_{2 n}\right)=q_{0}(0) \ldots q_{2 n}(0)$. Using any elements $q_{0}, q_{\alpha} \in$ ${ }^{d} \mathcal{W}$ and $\varphi \in{ }^{d} \mathcal{W}^{\otimes(n+n+1)}$, for $p_{0}=0$, we provide a $\mathrm{N}$-adapted generalization of the formula for $2 n$-th Hochschild cocycle [16],

$$
\begin{aligned}
& C^{n+n}\left({ }^{d} \mathcal{W},{ }^{d} \mathcal{W}^{*}\right) \ni \tau_{n+n}(\varphi)\left(q_{0}\right) \\
= & \mu_{2 n}\left(\int_{\Delta_{2 n}} \prod_{0 \leq \beta \leq \gamma \leq 2 n} e^{v\left(p_{\beta}-p_{\gamma}+1 / 2\right) b_{\beta \gamma}} \pi_{n+n}\left(q_{0} \otimes \varphi\right) \delta p_{1} \wedge \ldots \wedge \delta p_{2 n}\right),
\end{aligned}
$$

where $\pi_{n+n}(\varphi)\left(q_{0} \otimes \ldots \otimes q_{2 n}\right)=\varepsilon^{\alpha_{1} \ldots \alpha_{2 n}} q_{0} \otimes \frac{\delta q_{1}}{\partial z^{\alpha_{1}}} \otimes \ldots \otimes \frac{\delta q_{2 n}}{\partial z^{\alpha_{2 n}}}$ acts on respective tensor products of nonholonomic bases of type (5) and $\varepsilon^{\alpha_{1} \ldots \alpha_{2 n}}$ is absolutely antisymmetric. In the formula (13), the action of antisymmetric form $b$ on the $\alpha$-th and $\beta$-th components of ${ }^{d} \mathcal{W}^{\otimes(n+n+1)}$ and $q_{\gamma} \in{ }^{d} \mathcal{W}$ is denoted by

$$
b_{\alpha \beta}\left(q_{0} \otimes \ldots \otimes q_{2 n}\right)=b^{\alpha_{\gamma} \alpha_{\nu}}\left(q_{0} \otimes \ldots \otimes \frac{\delta q_{\gamma}}{\partial z^{\alpha_{\gamma}}} \otimes \ldots \otimes \frac{\delta q_{\nu}}{\partial z^{\alpha_{\nu}}} \otimes \ldots \otimes q_{2 n}\right) .
$$

Let us consider an associative algebra $\mathcal{A}$ with unit over a field $K$ with caracteristic zero and denote, for instance, by $\mathfrak{g l}_{k}\left({ }^{d} \mathcal{W}\right)$ the Lie algebra of $k \times k$ matrices with values in quadratic monomials in ${ }^{d} \mathcal{W}$. Also, we shall use matrix algebras of type $\mathfrak{g l}_{k}(\mathcal{A})$ and dual ones, with values in the dual module $\mathcal{A}^{*}$. Denoting by $C^{\bullet}\left(\mathcal{A}, \mathcal{A}^{*}\right)$ the Hochschild cochain complex with coefficients in the dual module $\mathcal{A}^{*}$ (for $\mathcal{A} \rightarrow \mathfrak{g l}_{k}(\mathcal{A})$, the similar complex is written $\left.C^{\bullet}\left(\mathfrak{g l}_{k}(\mathcal{A}), \mathfrak{g l}_{k}(\mathcal{A})^{*}\right)\right)$, we can construct a chain map

$$
{ }^{k} \phi: C^{\bullet}\left(\mathcal{A}, \mathcal{A}^{*}\right) \rightarrow C^{\bullet}\left(\mathfrak{g l}_{k}(\mathcal{A}), \mathfrak{g l}_{k}(\mathcal{A})^{*}\right)
$$

following the formula

$$
\begin{aligned}
& { }^{k} \phi(\psi)\left(A_{1} \otimes a_{1}, \ldots, A_{r} \otimes a_{r}\right)\left(A_{0} \otimes a_{0}\right) \\
= & \frac{1}{r !} \sum_{s \in S_{r}}(-1)^{s} \psi\left(a_{s(1)} \otimes \ldots \otimes a_{s(r)}\right)\left(a_{0}\right) \operatorname{tr}\left(A_{0} A_{s(1)} \ldots A_{s(r)}\right),
\end{aligned}
$$

where $S_{r}$ denotes the group of permutations of $r$ elements, matrices $A_{s} \in$ $\mathfrak{g l}_{k}(K), a_{s} \in \mathcal{A}$, and $\psi \in C^{r}\left(\mathcal{A}, \mathcal{A}^{*}\right)$. The action of map on cocycle (13) result in the $2 n$-th cocycle in the chain complex $C^{\bullet}\left(\mathfrak{g l}_{k}\left({ }^{d} \mathcal{W}\right), \mathfrak{g l}_{k}\left({ }^{d} \mathcal{W}\right)^{*}\right)$,

$$
{ }^{k} \Theta_{n+n}={ }^{k} \phi\left(\tau_{n+n}\right): \wedge^{2 n}\left(\mathfrak{g l}_{k}\left({ }^{d} \mathcal{W}\right)\right) \otimes \mathfrak{g l}_{k}\left({ }^{d} \mathcal{W}\right) \rightarrow \mathbb{C}((v)) .
$$


The cocycle (15) presents a N-adapted generalization of the results from [16, 12 for symplectic manifolds. In our case, we construct a trace density map for a quantum d-algebra of functions on almost Kähler manifolds induced by nonholonomic distributions on Lagrange and Einstein-Finsler spaces.

The Chern-Weil homomorphisms are defined for projections of Lie algebras to their subalgebras. N-adapted geometric constructions on nonholonomic manifolds are with associated Lie groups/algebras and related geometric maps are distinguished by the -connection structure. We call a Lie distinguished algebra (d-algebra) ${ }^{d} \mathfrak{g}={ }^{h} \mathfrak{g} \oplus{ }^{v} \mathfrak{g}=\left({ }^{h} \mathfrak{g},{ }^{v} \mathfrak{g}\right)$ a couple of conventional horizontal and vertical Lie algebras associated to a $\mathrm{N}$-connection splitting $T \mathbf{V}=h \mathbf{V} \oplus v \mathbf{V}$ (see also (10)). A Lie d-algebra ${ }^{d} \mathfrak{g}$ may have a dsubalgebra ${ }^{d} \rho \subset{ }^{d} \mathfrak{g}$, when ${ }^{d} \rho=\left({ }^{h} \rho \subset{ }^{h} \mathfrak{g},{ }^{v} \rho \subset{ }^{v} \mathfrak{g}\right)$. Let us suppose there is an ${ }^{h} \rho$-equivariant $\mathrm{N}$-adapted projection ${ }^{N} p r:\left({ }^{h} \mathfrak{g} \rightarrow{ }^{h} \rho,{ }^{v} \mathfrak{g} \rightarrow{ }^{v} \rho\right)$ satisfying the properties that the $h$ - and $v$-components of maps commute respectively with adjoint actions of ${ }^{h} \rho$ and ${ }^{v} \rho$ and $\left.{ }^{N} p r\right|_{d_{\rho}}=I d_{d_{\rho}}$. We can characterize ${ }^{N} p r$ by its curvature

$$
{ }^{d} C\left({ }^{1} \zeta,{ }^{2} \zeta\right):=\left[\operatorname{pr}\left({ }^{1} \zeta\right), \operatorname{pr}\left({ }^{2} \zeta\right)\right]-\operatorname{pr}\left(\left[{ }^{1} \zeta,{ }^{2} \zeta\right]\right) \in \operatorname{Hom}\left(\wedge^{2}{ }^{d} \mathfrak{g},{ }^{d} \rho\right),
$$

for ${ }^{1} \zeta=\left({ }_{h}^{1} \zeta,{ }_{v}^{1} \zeta\right)$ and ${ }^{2} \zeta=\left({ }_{h}^{2} \zeta,{ }_{v}^{2} \zeta\right)$ which in N-adapted form splits into $h-, v$-components of curvature,

$$
\begin{array}{lll}
{ }^{h} C\left({ }_{h}^{1} \zeta,{ }_{h}^{2} \zeta\right):= & {\left[\operatorname{pr}\left({ }_{h}^{1} \zeta\right), \operatorname{pr}\left({ }_{h}^{2} \zeta\right)\right]-\operatorname{pr}\left(\left[{ }_{h}^{1} \zeta,{ }_{h}^{2} \zeta\right]\right) \in \operatorname{Hom}\left(\wedge^{2}{ }^{h} \mathfrak{g},{ }^{h} \rho\right),} \\
{ }^{v} C\left({ }_{v}^{1} \zeta,{ }_{v}^{2} \zeta\right):= & {\left[\operatorname{pr}\left({ }_{v}^{1} \zeta\right), \operatorname{pr}\left({ }_{v}^{2} \zeta\right)\right]-\operatorname{pr}\left(\left[{ }_{v}^{1} \zeta,{ }_{v}^{2} \zeta\right]\right) \in H o m\left(\wedge^{2}{ }^{v} \mathfrak{g},{ }^{v} \rho\right) .}
\end{array}
$$

For any given adjoint invariant $\mathrm{d}-$-form

$$
{ }^{d} A=\left({ }^{h} A \in\left(\left(S^{r}{ }^{h} \rho\right)^{*}\right){ }^{h} \rho,{ }^{v} A \in\left(\left(S^{r}{ }^{v} \rho\right)^{*}\right){ }^{v} \rho\right),
$$

the formulas

$$
\begin{aligned}
& { }^{h} \chi\left({ }^{h} A\right)\left({ }_{h}^{1} \zeta_{s},{ }_{h}^{2} \zeta_{s}, \ldots,{ }_{h}^{2 r} \zeta_{s}\right)= \\
& \quad \frac{1}{(2 r) !} \sum_{s \in S_{2 r}}(-1)^{s}{ }^{h} A\left({ }^{h} C\left({ }_{h}^{1} \zeta_{s},{ }_{h}^{2} \zeta_{s}\right), \ldots,{ }^{h} C\left({ }_{h}^{2 r-1} \zeta_{s},{ }_{h}^{2 r} \zeta_{s}\right)\right), \\
& { }^{v} \chi\left({ }^{v} A\right)\left({ }_{v}^{1} \zeta_{s},{ }_{v}^{2} \zeta_{s}, \ldots,{ }_{v}^{2 r} \zeta_{s}\right)= \\
& \quad \frac{1}{(2 r) !} \sum_{s \in S_{2 r}}(-1)^{s}{ }^{v} A\left({ }^{v} C\left({ }_{v}^{1} \zeta_{s},{ }_{v}^{2} \zeta_{s}\right), \ldots,{ }^{v} C\left({ }_{v}^{2 r-1} \zeta_{s},{ }_{v}^{2 r} \zeta_{s}\right)\right)
\end{aligned}
$$

define a relative Lie d-algebra cocycle ${ }^{d} \chi\left({ }^{d} A\right)={ }^{h} \chi\left({ }^{h} A\right)+{ }^{v} \chi\left({ }^{v} A\right) \in$ $C^{2 r}\left({ }^{d} \mathfrak{g},{ }^{v} \rho\right)$. We say that such maps determine a Chern-Weil N-adapted homomorphism inducing also a map from the d-vector space $\left(\left(\begin{array}{ll}S^{d} & d\end{array}\right)^{*}\right)^{d} \rho$ to $H^{2 r}\left({ }^{d} \mathfrak{g},{ }^{d} \rho\right)$. Such constructions do not depend on the type of ${ }^{N} p r$ and/or $\mathrm{N}$-connection splitting. 
Taking $\quad{ }^{d} \mathfrak{g}=\mathfrak{g l}_{k}\left({ }^{d} \mathcal{W}\right)=\mathfrak{g l}_{k}(h \mathcal{W}) \oplus \mathfrak{g l}_{k}(v \mathcal{W}), \quad{ }^{d} \rho=\mathfrak{g l}_{k} \oplus \mathfrak{s p}_{n+n}$, when the Lie d-algebra $\mathfrak{s p}_{n+n}$ is realized as a subalgebra of scalar matrices in $\mathfrak{g l}_{k}\left({ }^{d} \mathcal{W}\right)$ with values in quadratic monomials in ${ }^{d} \mathcal{W}$, we can define the projection ${ }^{N} p r:{ }^{d} \mathfrak{g} \rightarrow{ }^{d} \rho$ following the formulas

$$
\begin{aligned}
& { }^{N} \operatorname{pr}(\zeta)={ }_{0}^{N} \operatorname{pr}(\zeta)+{ }_{2}^{N} \operatorname{pr}(\zeta), \\
& { }_{0}^{N} \operatorname{pr}(\zeta)=\zeta_{\mid z=0}, \quad{ }_{2}^{N} \operatorname{pr}(\zeta)=\frac{1}{k} \sigma_{2}(\operatorname{tr}(\zeta)) I_{k},
\end{aligned}
$$

where $I_{k}$ is the $k \times k$ identity matrix and $\sigma_{2}$ is the projection onto the monomials of second degree in $z$-variables.

An explicit computation (the original constructions are due to the FeiginFelder-Shoikhet theorem [16]) allows us to evaluate the N-adapted action of (15) on the identity matrix $I_{k} \in{ }^{d} \mathfrak{g}$. This provides the proof for

Theorem 3.1 There is a relative, with respect to distinguished subalgebra ${ }^{d} \rho=\mathfrak{g l}_{k} \oplus \mathfrak{s p}_{n+n}$, Lie d-algebra cocycle ${ }^{k} \Theta_{n+n} \in C^{n+n}\left({ }^{d} \mathfrak{g},{ }^{d} \mathfrak{g}^{*}\right)$ parametrized by $N$-adapted maps ${ }^{N} \varphi$ determined by (15), when

$$
C^{n+n}\left({ }^{d} \mathfrak{g},{ }^{d} \rho\right) \ni{ }^{N} \varphi={ }^{k} \Theta_{n+n}\left(\cdot, \ldots, \cdot, I_{k}\right): \wedge^{2 n}\left({ }^{d} \mathfrak{g}\right) \rightarrow \mathbb{C}((v)) .
$$

The cohomology class of such nonholonomic maps

$$
\left[{ }^{N} \varphi\right]=\left[{ }^{d} \chi\left({ }^{d} A_{r}\right)={ }^{h} \chi\left({ }^{h} A_{r}\right)+{ }^{v} \chi\left({ }^{v} A_{r}\right)\right]
$$

coincides with the image of the $r$-th component ${ }^{d} A_{r} \in\left(\left(S^{r}{ }^{d} \rho\right)^{*}\right){ }^{d} \rho$ of the adjoint invariant $d$-form ${ }^{d} A \in\left(\left(S^{d} \rho\right)^{*}\right)^{d} \rho$, when under nonholonomic Chern-Weil homomorphism (17)

$$
{ }^{d} A(\mathfrak{X}, \ldots, \mathfrak{X})=\operatorname{det}\left(\frac{{ }^{1} \mathfrak{X} / 2 v}{\sinh \left({ }^{1} \mathfrak{X} / 2 v\right)}\right)^{1 / 2} \operatorname{tr} \frac{{ }^{2} \mathfrak{X}}{v}
$$

for any $d$-vector $\mathfrak{X}$ with values of coefficients in the corresponding Lie $d_{-}$ group, $\mathfrak{X}={ }^{1} \mathfrak{X} \oplus{ }^{2} \mathfrak{X} \in \mathfrak{s p}_{n+n} \oplus \mathfrak{g l}_{k}$.

For integrable distributions on spaces under considerations, the results of this Theorem transform into "holonomic versions" studied in [16, 12].

\subsection{Fedosov quantization of Einstein-Finsler spaces}

Let ${ }^{\mathcal{L}} \mathbf{K}^{2 n}$ be an almost Kähler space derived for a N-anholonomic manifold $\mathbf{V}^{2 n}$. Deformation quantization of such spaces with nontrivial torsion can be performed following methods elaborated in Refs. [21, 28, 29, 30]. In this work, we revise the Fedosov quantization of Lagrange-Finsler and Einstein spaces in order to include in the scheme the bi-connection formalism which can be used for a perturbative model of quantum gravity [33]. 
We introduce the tensor $\Lambda^{\alpha \beta} \doteqdot \theta^{\alpha \beta}-i \mathbf{g}^{\alpha \beta}$, where $\theta^{\alpha \beta}$ is a form (9) where values with "up" indices are constructed using $\mathbf{g}^{\alpha \beta}$ being the inverse to $\mathbf{g}_{\alpha \beta}$ (17). Considering a nonholonomic vector bundle $\mathcal{E}$ of rank $k$ over ${ }^{\mathcal{L}} \mathbf{K}^{2 n}$, and $\mathbf{V}^{2 n}$, we denote by $\operatorname{End}(\mathcal{E})$ the bundle of endomorphisms of ${ }^{\mathcal{L}} \mathbf{K}^{2 n}$ and by $\operatorname{End} \mathbf{V}=\operatorname{Sec}\left({ }^{\mathcal{L}} \mathbf{K}^{2 n}, \operatorname{End}(\mathcal{E})\right)$ the d-algebra of global sections of $\operatorname{End}(\mathcal{E})$.

The formalism of deformation quantization can be developed by using $C^{\infty}\left({ }^{\mathcal{L}} \mathbf{K}^{2 n}\right)[[v]]$, the space of formal series of variable $v$ with coefficients from $C^{\infty}\left(\mathcal{L}_{\mathbf{K}^{2 n}}\right)$ on a Poisson manifold $\left({ }^{\mathcal{L}} \mathbf{K}^{2 n},\{\cdot, \cdot\}\right)$. In this work, we deal with the almost Poisson structure defined by the canonical almost symplectic structure. An associative d-algebra structure on $C^{\infty}\left({ }^{\mathcal{L}} \mathbf{K}^{2 n}\right)[[v]]$ can be defined canonically with a $v$-linear and $v$-adically continuous star product

$$
{ }^{1} f *{ }^{2} f=\sum_{r=0}^{\infty}{ }_{r} C\left({ }^{1} f,{ }^{2} f\right) v^{r},
$$

where ${ }_{r} C, r \geq 0$, are bilinear operators on $C^{\infty}\left({ }^{\mathcal{L}} \mathbf{K}^{2 n}\right)$, for ${ }_{0} C\left({ }^{1} f,{ }^{2} f\right)=$ ${ }^{1} f{ }^{2} f$ and ${ }_{1} C\left({ }^{1} f,{ }^{2} f\right)-{ }_{1} C\left({ }^{2} f,{ }^{1} f\right)=i\left\{{ }^{1} f,{ }^{2} f\right\} ; i$ being the complex unity. Such a $*$-operation defines a quantization the d-algebra $E n d_{\mathbf{V}}$, i.e. an associative $\mathbb{C}((v))$-linear product in $\operatorname{End}_{\mathbf{V}}((v))$, associated to a linear connection ${ }^{\mathcal{E}} \partial$ in $\mathcal{E}$, defined by a 1 -form ${ }^{\mathcal{E}} \boldsymbol{\Gamma}$ with coefficients in a vector space of dimension $k$. Considering any bi-differential operators ${ }_{r} Q: E_{n} d_{\mathbf{V}} \otimes E_{n} d_{\mathbf{V}} \rightarrow E_{n} d_{\mathbf{V}}$ such that, for any $a, b \in E_{n} d_{\mathbf{V}},{ }_{1} Q(a, b)-$ ${ }_{1} Q(b, a)=\theta^{\alpha \beta} \mathcal{E}_{\partial_{\alpha}}(a)^{\mathcal{E}} \partial_{\alpha}(b)$, we define a "total" star product (quantization of nonholonomic $\mathcal{E}$ ) as

$$
a * b=a b+\sum_{r=1}^{\infty}{ }_{r} Q(a, b) v^{r} .
$$

The product (20) is used for constructing a formal Wick product

$$
\left.a \circ b(z) \doteqdot \exp \left(i \frac{v}{2} \boldsymbol{\Lambda}^{\alpha \beta} \frac{\partial^{2}}{\partial z^{\alpha} \partial{ }^{1} z^{\beta}}\right) a(z) b\left({ }^{1} z\right)\right|_{z={ }^{1} z},
$$

for two elements $a$ and $b$ defined by series of type

$$
a(v, u, z)=\sum_{r \geq 0,|\{\alpha\}| \geq 0} a_{r,\{\alpha\}}(u) z^{\{\alpha\}} v^{r},
$$

where by $\{\alpha\}$ we label a multi-index. In terms of $\mathrm{N}$-elongated derivatives, the product (21) can be written similarly to (11) for the Weyl d-algebra ${ }^{d} \mathcal{W}$. In (22), $z^{\alpha}$ are fiber coordinates of the tangent bundle $T{ }^{\mathcal{L}} \mathbf{K}^{2 n} \simeq$ $T \mathbf{V}^{2 n}$ and $a_{r,\{\alpha\}}(u)=a_{r,{ }^{1} \alpha^{2} \alpha \ldots{ }^{l} \alpha}(u)$ can be represented as sections of $\operatorname{End}(\mathcal{E}) \otimes S^{l}\left(T^{*} \mathbf{V}^{2 n}\right)$.

The formulas (201)-(22) define a formal Wick algebra $\mathbf{W}_{u}$ associated with the tangent space $T_{u} \mathbf{V}^{2 n}$, for $u \in \mathbf{V}^{2 n}$. The fibre product (21) can be trivially 
extended to the space of $\mathbf{W}$-valued $\mathrm{N}$-adapted differential forms $\mathcal{W} \otimes \Lambda$ by means of the usual exterior product of the scalar forms $\Lambda$, where $\mathcal{W}$ denotes the sheaf of smooth sections of $\mathbf{W}$. There is a standard grading on $\Lambda$ denoted $\operatorname{deg}_{a}$. We can introduce gradings $\operatorname{deg}_{v}, \operatorname{deg}_{s}, \operatorname{deg}_{a}$ on $\mathcal{W} \otimes \Lambda$ defined on homogeneous elements $v, z^{\alpha}$ and $\mathbf{e}^{\alpha}(5)$ as follows: $\operatorname{deg}_{v}(v)=1$, $\operatorname{deg}_{s}\left(z^{\alpha}\right)=1, \operatorname{deg}_{a}\left(\mathbf{e}^{\alpha}\right)=1$, and all other gradings of the elements $v, z^{\alpha}, \mathbf{e}^{\alpha}$ are set to zero. The product $\circ$ from (21) on $\mathcal{W} \otimes \boldsymbol{\Lambda}$ is bigraded. This is written w.r.t the grading $D e g=2 \operatorname{deg}_{v}+\operatorname{deg}_{s}$ and the grading $\operatorname{deg}_{a}$. The filtration (12) of the Weyl d-algebra gives also a natural filtration of the nonholonomic Weyl d-algebra bundle $\mathbf{W}(E n d \mathbf{v})$ whose sections are the formal power series (22). The $\mathrm{N}$-connection structure on $T \mathbf{V}^{2 n}$ determines also $\mathrm{h}-$ and $\mathbf{v}$-splitting with filtrations in $h \mathbf{W}\left(E n d_{\mathbf{V}}\right)$ and $v \mathbf{W}\left(E n d_{\mathbf{V}}\right)$. For holonomic configurations the algebraic and topological properties are examined in [12].

In what follows we shall use the d-algebra ${ }^{d} \boldsymbol{\Omega}^{\bullet}\left({ }^{\mathcal{L}} \mathbf{K}^{2 n}\right)$ of exterior dforms on ${ }^{\mathcal{L}} \mathbf{K}^{2 n}$ as an d-algebra embedded into ${ }^{d} \boldsymbol{\Omega}^{\bullet}(\mathbf{W}(E n d \mathbf{V}))$. For any exterior $\mathrm{d}$-form $\varpi \in{ }^{d} \boldsymbol{\Omega}^{\bullet}\left({ }^{\mathcal{L}} \mathbf{K}^{2 n}\right)$, the is a map sending it into the scalar matrix $\varpi I_{k} \in{ }^{d} \boldsymbol{\Omega}^{\bullet}\left(\mathbf{W}\left(E n d_{\mathbf{V}}\right)\right)$. This natural map, $i:{ }^{d} \boldsymbol{\Omega}^{\bullet}\left({ }^{\mathcal{L}} \mathbf{K}^{2 n}\right) \rightarrow$ ${ }^{d} \boldsymbol{\Omega}^{\bullet}\left(\mathbf{W}\left(E n d_{\mathbf{V}}\right)\right)$, defines the embedding of $\mathrm{d}$-forms.

We consider a d-connection $\mathbf{D}$ on $T \mathbf{V}^{2 n}$ which is compatible to the symplectic d-form $\theta^{\alpha \beta}$ (9), $\mathbf{D} \theta=0$, and a connection ${ }^{\mathcal{E}} \partial$ on $\mathcal{E}$. There is a linear d-operator ${ }^{d} \mathcal{D}:{ }^{d} \boldsymbol{\Omega}^{\bullet}\left(\mathbf{W}\left(E n d_{\mathbf{V}}\right)\right) \rightarrow{ }^{d} \boldsymbol{\Omega}^{\bullet+1}(\mathbf{W}(E n d \mathbf{V}))$, i.e.

$$
{ }^{d} \mathcal{D}=\delta u^{\alpha}\left(\mathbf{e}_{\alpha}-\boldsymbol{\Gamma}_{\beta \gamma}^{\alpha}(u) z^{\gamma} \frac{\delta}{\partial z^{\alpha}}\right)+\left[{ }^{\mathcal{E}} \boldsymbol{\Gamma}, \cdot\right]
$$

where $\boldsymbol{\Gamma}_{\beta \gamma}^{\alpha}$ are coefficients of $\mathbf{D}$ and ${ }^{\mathcal{E}} \boldsymbol{\Gamma}$ is the connection form of ${ }^{\mathcal{E}} \partial$. The curvature and torsion of such operators, for instance, for $\mathbf{D}=\widehat{\mathbf{D}} \equiv{ }_{\theta} \widehat{\mathbf{D}}$, see (10) is computed in [28, 29, 30]. In our case, there is an additional term defined by ${ }^{\mathcal{E}} \boldsymbol{\Gamma}$ but this does not modify substantially the properties of ${ }^{d} \mathcal{D}$ determined by canonical Lagrange-Finsler $\mathrm{d}$-connections. We shall write ${ }^{d} \widehat{\mathcal{D}}$ if such a connection is induced by ${ }_{\theta} \widehat{\mathbf{D}}$.

Definition 3.2 A Fedosov-Finsler normal d-connection ${ }_{r}^{d} \mathcal{D}$ is a nilpotent $N$-adapted derivation of the graded $d$-algebra,

$$
{ }_{r}^{d} \mathcal{D}={ }^{d} \mathcal{D}+v^{-1}\left[{ }^{\theta} r, \cdot\right],
$$

for ${ }^{\theta} r=z^{\alpha} \theta_{\alpha \beta}(u) \delta u^{\beta}+r$, where $r$ is an element in the set of $d$-forms ${ }^{d} \boldsymbol{\Omega}^{1}(\mathbf{W}(E n d \mathbf{V})$.

Any derivations of a d-connection $\mathbf{D}$ and a connection ${ }^{\mathcal{E}} \partial$ can be absorbed into a d-form $r \in{ }^{d} \boldsymbol{\Omega}^{1}\left(\mathbf{W}\left(E n d_{\mathbf{V}}\right)\right.$. Two potentials ${ }^{\mathcal{E}} \boldsymbol{\Gamma}$ and ${ }^{\mathcal{E}} \widetilde{\boldsymbol{\Gamma}}$ result in equivalent Fedosov-Finsler d-connections (24) if

$$
{ }^{\mathcal{E}} \widetilde{\boldsymbol{\Gamma}}=\mathbf{B}^{-1} \circ\left({ }^{\mathcal{E}} \boldsymbol{\Gamma} \circ \mathbf{B}+v^{d} \mathcal{D}\right),
$$


where $\mathbf{B}$ belongs to the affine subspace $I_{k} \oplus \operatorname{Sec}\left(\mathbf{W}^{1}\left(E n d_{\mathbf{V}}\right)\right)$ in

$\operatorname{Sec}\left(\mathbf{W}\left(E n d_{\mathbf{V}}\right)\right)$ consisting of the sums $\mathbf{B}=\mathbf{I}_{k}+\mathbf{B}_{1}$, for an arbitrary dvector $\mathbf{B}_{1} \in \operatorname{Sec}\left(\mathbf{W}^{1}(E n d \mathbf{V})\right)$ distinguished by $\mathrm{N}$-connection. This results in equality

$$
{ }_{r}^{d} \widetilde{\mathcal{D}}={ }_{r}^{d} \mathcal{D}+\left[\mathbf{B}^{-1} \circ{ }_{r}^{d} \mathcal{D} \mathbf{B}, \cdot\right]
$$

For a trivial connection ${ }^{\mathcal{E}} \partial$, Fedosov-Finsler normal d-connections are completely determined by fundamental geometric objects on ${ }^{\mathcal{L}} \mathbf{K}^{2 n}$. The normal d-connection $\widehat{\mathbf{D}}=\left\{\widehat{\boldsymbol{\Gamma}}_{\alpha \beta}^{\gamma}\right\}$ (10) can be extended to the d-operator

$$
\widehat{\mathbf{D}}(a \otimes \lambda) \doteqdot\left(\mathbf{e}_{\alpha}(a)-u^{\beta} \widehat{\boldsymbol{\Gamma}}_{\alpha \beta}^{\gamma} z^{z} \mathbf{e}_{\alpha}(a)\right) \otimes\left(\mathbf{e}^{\alpha} \wedge \lambda\right)+a \otimes d \lambda,
$$

on $\mathcal{W} \otimes \Lambda$, where ${ }^{z} \mathbf{e}_{\alpha}$ is $\mathbf{e}_{\alpha}$ (5) redefined in $z$-variables.

Definition 3.3 The Fedosov d-operators $\delta$ and $\delta^{-1}$ on $\mathcal{W} \otimes \boldsymbol{\Lambda}$ are

$$
\delta(a)=\mathbf{e}^{\alpha} \wedge{ }^{z} \mathbf{e}_{\alpha}(a), \text { and } \delta^{-1}(a)=\left\{\begin{array}{c}
\frac{i}{p+q} z^{\alpha} \mathbf{e}_{\alpha}(a), \text { if } p+q>0, \\
0, \text { if } p=q=0,
\end{array}\right.
$$

where any $a \in \mathcal{W} \otimes \boldsymbol{\Lambda}$ is homogeneous w.r.t. the grading $\operatorname{deg}_{s}$ and $\operatorname{deg}_{a}$ with $\operatorname{deg}_{s}(a)=p$ and $\operatorname{deg}_{a}(a)=q$.

The d-operators (27) define a N-adapted map,

$$
a=\left(\delta \delta^{-1}+\delta^{-1} \delta+\sigma\right)(a),
$$

where $a \longmapsto \sigma(a)$ is the projection on the $\left(\operatorname{deg}_{s}, \operatorname{deg}_{a}\right)$-bihomogeneous part of $a$ of degree zero, $\operatorname{deg}_{s}(a)=\operatorname{deg}_{a}(a)=0 ; \delta$ is also a $\operatorname{deg}_{a}$-graded derivation of the d-algebra $(\mathcal{W} \otimes \boldsymbol{\Lambda}, \circ)$.

The Fedosov-Finsler normal d-connection ${ }_{r}^{d} \mathcal{D}(24)$ can written using the $\mathrm{d}$-operator $\delta$, treated as the Koszul derivation of the d-algebra

${ }^{d} \boldsymbol{\Omega}^{\bullet}\left(\mathbf{W}\left(E n d_{\mathbf{V}}\right)\right)$. The corresponding Koszul N-adapted differential

$$
\delta^{-1}(a)=z^{\alpha} \iota \mathbf{e}_{\alpha} \int_{0}^{1} a(u, v, \tau z, \tau \delta u) \frac{d \tau}{\tau},
$$

where the symbol $\iota \mathbf{e}_{\alpha}$ is used for the contraction of an exterior d-form with d-vector $\mathbf{e}_{\alpha}$, for $\delta^{-1}$ extended to $\operatorname{Sec}(\mathbf{W}(E n d \mathbf{v}))$ by zero. Using the doperator (28), we can verify that such a $\delta^{-1}$ is really a homotopy d-operator for $\delta$. Really, we have that for any $a \in{ }^{d} \boldsymbol{\Omega}\left(\mathbf{W}\left(E n d_{\mathbf{V}}\right)\right)$ we can express

$$
a=\sigma(a)+\delta \delta^{-1} a+\delta^{-1} \delta a,
$$

where $\sigma$ is a $\mathrm{N}$-adapted natural projection

$$
\sigma(a)=a_{\mid z=0, \delta u=0},
$$

when $a \in{ }^{d} \boldsymbol{\Omega}^{\bullet}\left(\mathbf{W}\left(E n d_{\mathbf{V}}\right)\right)$, i.e. it is defined a map of ${ }^{d} \boldsymbol{\Omega}^{\bullet}(\mathbf{W}(E n d \mathbf{V}))$ onto the d-algebra of endomorphisms $\operatorname{End}_{\mathbf{V}}(v)$ preserving $h$ - and $v$-splitting. 
Theorem 3.2 Any (pseudo) Lagrange-Finsler/Riemanian metric $\mathbf{g}$ (7) defines a flat normal Fedosov d-connection

$$
\widehat{\mathcal{D}} \doteqdot-\delta+\widehat{\mathbf{D}}-\frac{i}{v} a d_{W i c k}(r)
$$

satisfying the condition $\widehat{\mathcal{D}}^{2}=0$, where the unique element $r \in \mathcal{W} \otimes \boldsymbol{\Lambda}$, $\operatorname{deg}_{a}(r)=1, \delta^{-1} r=0$, solves the equation

$$
\delta r=\widehat{\mathcal{T}}+\widehat{\mathcal{R}}+\widehat{\mathbf{D}} r-\frac{i}{v} r \circ r
$$

and this element can be computed recursively with respect to the total degree Deg as follows:

$$
\begin{aligned}
r^{(0)} & =r^{(1)}=0, r^{(2)}=\delta^{-1} \widehat{\mathcal{T}}, r^{(3)}=\delta^{-1}\left(\widehat{\mathcal{R}}+\widehat{\mathbf{D}} r^{(2)}-\frac{i}{v} r^{(2)} \circ r^{(2)}\right), \\
r^{(k+3)} & =\delta^{-1}\left(\widehat{\mathbf{D}} r^{(k+2)}-\frac{i}{v} \sum_{l=0}^{k} r^{(l+2)} \circ r^{(l+2)}\right), k \geq 1,
\end{aligned}
$$

where by $a^{(k)}$ we denote the Deg-homogeneous component of degree $k$ of an element $a \in \mathcal{W} \otimes \boldsymbol{\Lambda}$.

Proof. Similarly to constructions provided in [14, 15, 21], using Fedosov's d-operators (27) [28, 29, 30], we verify the conditions of the theorem. Straightforward verification of the property $\widehat{\mathcal{D}}^{2}=0$, with formal series of type (22), for $r$, and can be performed in N-adapted form for $\widehat{\mathbf{D}}$, with torsion $\widehat{\mathcal{T}}$, A.3), and curvature, $\widehat{\mathcal{R}}$ (A.4).

The conditions of this Theorem can be redefined for a nonholonomic vector bundle $\mathcal{E}$ of rank $k$ over ${ }^{\mathcal{L}} \mathbf{K}^{2 n}$. The $\mathrm{N}$-adapted constructions are similar for $h$ - and $v$-components provided in section 5.3 of [12] and Theorem 2 and Remark 2 in [15]. We summarize four necessary statements in

Corollary 3.1-Remarks: For a normal d-connection $\widehat{\mathbf{D}} \equiv{ }_{\theta} \widehat{\mathbf{D}}(10)$ on ${ }^{\mathcal{L}} \mathbf{K}^{2 n}$, a connection ${ }^{\mathcal{E}} \partial$ in a nonholonomic vector bundle $\mathcal{E}$ of rank $k$ over ${ }^{\mathcal{L}} \mathbf{K}^{2 n}$ and ${ }^{v} \Omega$ is a series of closed distinguished (by $N$-connections) twoforms in $v \Omega^{2}\left({ }^{\mathcal{L}} \mathbf{K}^{2 n}\right)$. We can derive from Theorem 3.2 the following

1. There is a nilpotent $N$-adapted derivation ${ }_{r}^{d} \widehat{\mathcal{D}}={ }^{d} \widehat{\mathcal{D}}+v^{-1}\left[r-\theta_{\alpha \beta}(u) z^{\beta} \mathbf{e}^{\alpha}, \cdot\right] \quad$ (determined respectively as $/$ by (24), (9) and (6)) ) when the distinguished Fedosov-Weyl curvature

$$
\begin{aligned}
{ }^{W} \widehat{\mathcal{C}} & =v\left(\widehat{\mathcal{R}}+{ }^{V} \mathcal{R}\right)+2{ }^{d} \mathcal{D}\left({ }^{\mathcal{E}} \boldsymbol{\Gamma}\right)+v^{-1}\left[{ }^{\mathcal{E}} \boldsymbol{\Gamma},{ }^{\mathcal{E}} \boldsymbol{\Gamma}\right] \\
& =-\theta+{ }^{v} \Omega,
\end{aligned}
$$

where ${ }^{V} \mathcal{R}$ is the d-curvature of ${ }^{\mathcal{E}} \boldsymbol{\Gamma},{ }^{d} \mathcal{D}$ is given by (23) and the element $r \in{ }^{d} \boldsymbol{\Omega}^{1}\left(\mathbf{W}^{2}\left(\right.\right.$ End $\left.\left._{\mathbf{V}}\right)\right)$ satisfies the condition $\delta^{-1} r=0$. 
2. A star product (20) in End $\mathbf{V}((v))$ is induced via a d-vector space isomorphism

$$
\chi: \operatorname{End}_{\mathbf{V}}((v)) \simeq \widehat{\mathbf{D}}_{S e c}\left(\mathbf{W}\left(\operatorname{End}_{\mathbf{V}}\right)\right)
$$

from $\operatorname{End}_{V}((v))$ to the d-algebra $\widehat{\mathbf{D}}_{S e c}\left(\mathbf{W}\left(E_{n} d_{\mathbf{V}}\right)\right)$ of flat sections of $\mathbf{W}\left(E_{\text {End }}\right)$ with respect to the Fedosov-Finsler normal d-connection ${ }_{r}^{d} \widehat{\mathcal{D}}$ (24).

3. A d-connection ${ }_{r}^{d} \widehat{\mathcal{D}}$ is equivalent in the sence (25) to any ${ }_{r}^{d} \mathcal{D}$ when respective distinguished Fedosov-Weyl curvatures (31), ${ }^{W} \widehat{\mathcal{C}}$ and ${ }^{W} \mathcal{C}$, represent the same de Rham cohomology class in $H_{D R}^{2}\left({ }^{\mathcal{L}} \mathbf{K}^{2 n}\right)[[v]]$.

4. For a trivial $d$-vector bundle of rank $k=1$, the star product (20) and isomorphism $\chi(32)$ result in the star product (19) when the cohomology class of ${ }^{W} \widehat{\mathcal{C}}$ (31) characterizes the star product in $C\left({ }^{\mathcal{L}} \mathbf{K}^{2 n}\right)((v))$. Here we emphasize that there are two classes of equivalence, for the $h$ - and $v$-components.

For simplicity, we provided N-adapted constructions only for the base nonolonomic manifold ${ }^{\mathcal{L}} \mathbf{K}^{2 n}$ of a vector bundle $\mathcal{E}$. In general, a $\mathrm{N}$-connection structure can be considered for the tangent space $T \mathcal{E}$ (we omit such considerations in this work).

\section{Algebraic Index Theorem for Lagrange and Einstein-Finsler Spaces}

Various versions and modifications of the algebraic index theorem generalize the Atiyah-Singer theorem [6] from the case of a cotangent bundle to arbitrary symplectic and Poisson manifolds [8, 10, 12, 26]. Generalizations were also considered for nonholonomic manifolds and Lagrange-Finsler and Einstein gerbes [36]. In this section, we apply N-adapted techniques to provide a local index theorem for almost Kähler models of Lagrange-Finser and Einstein spaces.

\section{1 $\mathrm{N}$-adapted trace density maps}

We use the Feigin-Felder-Shoikhet (FFS) method [16] in order to construct a natural, in our case, $\mathrm{N}$-adapted density map. For vector bundles on symplectic manifolds such maps were considered for a proof of the local algebraic index theorem [12]. In our approach, the constructions should be conventionally dubbed for $h$ - and $v$-components of geometric objects distinguished by $\mathrm{N}$-connection structure. 
Definition 4.1 A $N$-adapted (i.e. preserving a Whitney sum $T{ }^{\mathcal{L}} \mathbf{K}^{2 n}=$ $h^{\mathcal{L}} \mathbf{K}^{2 n} \oplus v{ }^{\mathcal{L}} \mathbf{K}^{2 n}$ of type (11)) $\mathbb{C}$-linear nonholonomic map

$$
{ }_{N}^{d} \operatorname{tr}: \operatorname{End}_{\mathbf{V}}((v)) \rightarrow H^{2 n^{\prime}}\left(\mathbf{K}^{2 n}\right)((v))
$$

vanishing on commutators ${ }_{N}^{d} \operatorname{tr}(a * b-b * a)=0$, defined by star $d$-operator (20) for any $a, b \in \operatorname{End}_{\mathbf{V}}((v))$, is called a trace density d-map.

In the above formula for ${ }_{N}^{d} t r$, we write $H^{2 n^{\prime}}$ with a prime index in order to avoid confusions with the dimension $n$ in $\mathbf{K}^{2 n}$.

Using the nonholonomic cocycle ${ }^{k} \Theta_{n+n}$ (15) of d-group ${ }^{d} \mathfrak{g}=\mathfrak{g l}_{k}\left({ }^{d} \mathcal{W}\right)=$ $\mathfrak{g l}_{k}(h \mathcal{W}) \oplus \mathfrak{g l}_{k}(v \mathcal{W})$ relative to N-adapted "shifts" ${ }^{d} \rho=\mathfrak{g l}_{k} \oplus \mathfrak{s p}_{n+n}$, we can

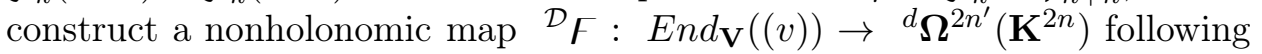
the formula

$$
{ }^{\mathcal{D}} \digamma(a)=v^{-n k} \Theta_{n+n}\left({ }^{\theta} r, \ldots,{ }^{\theta} r, \chi(a)\right),
$$

where ${ }^{\theta} r \in{ }^{d} \boldsymbol{\Omega}^{1}(\mathbf{W}(E n d \mathbf{V}))$ is the distinguished 1-form considered in the formula for the Fedosov-Finsler d-connection ${ }_{r}^{d} \mathcal{D}(24)$ and $\chi(a)$ is the isomorphism (32).

Let us consider respectively two nonholonomic vector bundles ${ }^{1} \mathcal{E}$ and ${ }^{2} \mathcal{E}$ of rank $k$ over ${ }^{\mathcal{L}} \mathbf{K}^{2 n}$ endowed with Fedosov-Finsler d-connections ${ }_{r}^{d} \widehat{\mathcal{D}}_{1}$ and ${ }_{r}^{d} \widehat{\mathcal{D}}_{2}$ on $\mathbf{W}\left(\right.$ End $\left.^{1} \mathbf{V}\right)$ and $\mathbf{W}\left(\right.$ End $\left.^{2} \mathbf{V}\right)$; elements ${ }^{1} a \in E n d^{1} \mathbf{V}$ and a pair of $\mathrm{N}$-adapted endomorphisms $a, b \in \operatorname{End}_{\mathbf{V}}((v))$ corresponding to $\mathcal{E}$ over ${ }^{\mathcal{L}} \mathbf{K}^{2 n}$; two equivalent d-operators ${ }^{\mathcal{E}} \widetilde{\boldsymbol{\Gamma}}$ and ${ }^{\mathcal{E}} \boldsymbol{\Gamma}$ in the sense of (25) with $\mathbf{B} \in I_{k} \oplus \operatorname{Sec}\left(\mathbf{W}^{1}\left(E n d_{\mathbf{V}}\right)\right)$ and corresponding equivalent Fedosov-Finsler doperators ${ }_{r}^{d} \mathcal{D}$ and ${ }_{r}^{d} \widetilde{\mathcal{D}}$; a N-adapted projection $\sigma$ (30) and isomorphism $\chi$ (32); the $r$-th component ${ }^{d} A_{r} \in\left(\left(S^{r} d^{d} \rho\right)^{*}\right)^{d} \rho$; the identity endomorphism $I_{k} \in E n d_{\mathbf{V}}$; we also consider curvature ${ }^{d} C\left({ }^{1} \zeta,{ }^{2} \zeta\right)$ (16) and projection (18). Applying respectively the $\mathrm{N}$-adapted constructions for ${ }^{k} \Theta_{n+n}$ in section 3.1 (see formulas (13) - (15) and Theorem 3.1) and dubbing for $h-v$-components the respective computations from [16] we prove

Theorem 4.1 The nonholonomic map ${ }^{D_{\mathcal{F}}}$ (33) is characterized by properties that ${ }^{\mathcal{D}_{1} \oplus \mathcal{D}_{2}} \digamma\left({ }^{1} a \oplus 0\right)={ }^{\mathcal{D}_{1}} \digamma\left({ }^{1} a\right)$ and three classes of $N$-adapted forms ${ }^{\mathcal{D}} \digamma(a * b-b * a),{ }^{\tilde{D}_{\digamma}} \digamma\left(\sigma\left(\mathbf{B}^{-1} \circ \chi\left({ }^{1} a\right) \circ \mathbf{B}\right)\right)-{ }^{\mathcal{D}} \boldsymbol{\digamma}\left({ }^{1} a\right)$ and ${ }^{\mathcal{D}} \digamma\left(I_{k}\right)-v^{n^{\prime}}$ ${ }^{d} A_{n^{\prime}}\left({ }^{d} C\left({ }^{\theta} r,{ }^{\theta} r\right), \ldots,{ }^{d} C\left({ }^{\theta} r,{ }^{\theta} r\right)\right)$ are of type $\delta^{d} \boldsymbol{\Omega}^{2 n^{\prime}-1}\left(\mathbf{K}^{2 n}\right)((v))$, for 0 denoting the trivial endomorphysm of ${ }^{2} \mathcal{E}$ and $\delta$ being the de Rham differential computed in $N$-adapted form.

From this theorem, we get

Corollary 4.1 We define a $N$-adapted trace density map

$$
{ }_{N}^{d} \operatorname{tr}(a)=\left[{ }^{\mathcal{D}} \digamma(a)\right]: \operatorname{End}_{\mathbf{V}}((v)) \rightarrow H^{2 n^{\prime}}\left(\mathbf{K}^{2 n}\right)((v))
$$

if the star-product $*$ in End $\mathbf{V}$, see (20), is determined by an isomorphism $\chi$ (32) and a normal d-connection $\widehat{\mathbf{D}} \equiv{ }_{\theta} \widehat{\mathbf{D}}(10)$. 
Finally, we note that for holonomic structures and vanishing distortion tensor (A.9) the formula (34) transform into the so-called FFS trace density map, see details in [16] and section 2 of [12].

\subsection{Main result: the nonholonomic algebraic index theorem}

We conclude our constructions on nonholonomic deformation quantization and N-adapted index theorem for an almost Kähler space $\mathcal{L}_{\mathbf{K}}{ }^{2 n}$ defined by a regular $\mathcal{L}$ on a $\mathrm{N}$-anholonomic manifold $\mathbf{V}^{2 n}$. Let $*$ be a star product (19) in the d-vector space $C\left({ }^{\mathcal{L}} \mathbf{K}^{2 n}\right)((v))$ of smooth functions on ${ }^{\mathcal{L}} \mathbf{K}^{2 n}$, with induced (20) following conditions of the statement 2 in Corollary 3.1 via the isomorphism $\chi(32)$. We consider an idempotent ${ }^{p} \zeta$ in the matrix $\mathrm{d}$-algebra ${ }^{d} \mathfrak{g}=\mathfrak{g l}_{k}\left({ }_{c}^{+} \mathcal{A}\right)$, where the subalgebra ${ }_{c}^{+} \mathcal{A}=C\left({ }^{\mathcal{L}} \mathbf{K}^{2 n}\right)[[v]] \subset{ }_{c} \mathcal{A}=C\left({ }^{\mathcal{L}} \mathbf{K}^{2 n}\right)((v))$, and use the same symbol ${ }_{r}^{d} \mathcal{D}$ for the naturally extended Fedosov-Finsler d-connection on the Weyl dalgebra bundle $\mathbf{W}\left(\operatorname{End}\left(l_{k}\right)\right)$ associated with the trivial bundle $l_{k}$ of rank $k$. At the next step, we assign to ${ }^{p} \zeta$ the top degree de Rham cohomology class

$$
\operatorname{cl}\left({ }^{p} \zeta\right)=\left[{ }^{\mathcal{D}} \digamma\left({ }^{p} \zeta\right)\right] \in H^{2 n^{\prime}}\left(\mathbf{K}^{2 n}\right)((v)) .
$$

For the $K_{0}$-group of the d-algebra ${ }_{c}^{+} \mathcal{A}$ to $H^{2 n^{\prime}}\left(\mathbf{K}^{2 n}\right)((v))$ we get $c l$ : $K_{0}\left({ }_{c}^{+} \mathcal{A}\right) \rightarrow H^{2 n^{\prime}}\left(\mathbf{K}^{2 n}\right)((v))$ following statements of Theorem 4.1.

Certain properties of a Fedosov quantized Lagrange-Finsler/ Einstein geometric model are characterized by star product (19), and (20), determined by the principal part of idempotents of $\mathfrak{g l}_{k}\left({ }_{c}^{+} \mathcal{A}\right)$.

Proposition 4.1 -Definition. The principal part ${ }^{0} \zeta$ of an idempotent ${ }^{p} \zeta \in \mathfrak{g l}_{k}\left({ }_{c}^{+} \mathcal{A}\right)$ is determined by the zeroth term ${ }^{0} \zeta={ }^{p} \zeta_{\mid v=0}$. There are two important properties for such principal parts:

1. Any idempotend in the matrix d-algebra $\mathfrak{g l}_{k} C\left({ }^{\mathcal{L}} \mathbf{K}^{2 n}\right)$ combined with the operation of taking the principal part gives a well-defined principal symbol map

$$
\mathcal{K}: K_{0}\left({ }_{c}^{+} \mathcal{A}\right) \rightarrow K_{0}\left({ }^{\mathcal{L}} \mathbf{K}^{2 n}\right) .
$$

2. If two idempotents ${ }^{p} \zeta_{1},{ }^{p} \zeta_{2} \in\left(\operatorname{End}_{V}[[v]], *\right)$, for a vector bundle $\mathcal{E}$ of rank $k$ over ${ }^{\mathcal{L}} \mathbf{K}^{2 n}$ and a star product $*$ (20) defined by Fedosov procedure, have equal principal parts, ${ }^{p} \zeta_{1 \mid v=0}={ }^{p} \zeta_{2 \mid v=0}$, then we get coincidence of cohomology classes, ${ }^{\mathcal{D}} \boldsymbol{\digamma}\left({ }^{p} \zeta_{1}\right)={ }^{\mathcal{D}} \boldsymbol{F}\left({ }^{p} \zeta_{2}\right)$.

Proof. The first statement above follows from a result in [9], that for any idempotent $\zeta$ in the matrix algebra $\mathfrak{g l}_{k}(C(\mathcal{M})$ ), for a symplectic manifold $\mathcal{M}$, we can define an idempotent $\zeta^{\prime}$ in $\mathfrak{g l}_{k}(C(\mathcal{M})[[v]], *)$, where $(C(\mathcal{M})[[v]], *)$ is a subalgebra of $(C(\mathcal{M})((v)), *)$, with a given star product $*$, when the principal part of $\zeta^{\prime}$ is $\zeta$. In our approach, we work with 
N-adapted structures on $C\left({ }^{\mathcal{L}} \mathbf{K}^{2 n}\right)$ and have to consider for $h$ - and $v$ components and almost symplectic d-connection $\widehat{\mathbf{D}} \equiv{ }_{\theta} \widehat{\mathbf{D}}(\underline{10})$ on $\mathcal{L}_{\mathbf{K}}{ }^{2 n}$ the explicit formula for principal parts in [15]. The second statement is a straightforward generalization for ${ }^{\mathcal{L}} \mathbf{K}^{2 n}$ of the proof of theorem 6.1.3 in the just mentioned Fedosov's monograph.

Theorem 4.2 The cohomology class $c l\left({ }^{0} k\right)$ for any element ${ }^{0} k \in K_{0}\left({ }_{c}^{+} \mathcal{A}\right)$ coincides with the top component of the cup product

$$
\operatorname{cl}\left({ }^{0} k\right)=\left[\widehat{A}\left({ }^{\mathcal{L}} \mathbf{K}^{2 n}\right) e^{-v^{-1}{ }^{W} \widehat{\mathcal{C}}} \operatorname{ch}\left(\mathcal{K}\left({ }^{0} k\right)\right)\right]_{n+n},
$$

where ch $\left(\mathcal{K}\left({ }^{0} k\right)\right)$ is the the Chern character of the principal symbol $\mathcal{K}\left({ }^{0} k\right)$ (35) of ${ }^{0} k$, the exponent is determined by ${ }^{W} \widehat{\mathcal{C}}=-\theta+{ }^{v} \Omega$ (31) (in the holonomic case it transforms into the so-called Deligne-Fedosov class) here computed by the star-product in ${ }_{c}^{+} \mathcal{A}$; we also consider in (36) the so-called $\widehat{A}$-genus of ${ }^{\mathcal{L}} \mathbf{K}^{2 n}$.

We sketch a proof of this theorem in Appendix B.

\subsection{Example: index encoding of Einstein-Finsler spaces}

It is possible to find explicit relations between the nonholonomic versions of the local index theorem and classification of exact solutions for Einstein and Einstein-Finsler classical and quantum (in the sense of deformation quantization) gravity. For instance, a very important mathematical and physical problem is that to formulate some well established criteria when a nonholonomical distinguished 1 -form $\theta$ (9) and related normal d-connection $\widehat{\mathbf{D}} \equiv{ }_{\theta} \widehat{\mathbf{D}}(10)$, via the Fedosov-Finsler normal d-connection ${ }_{r}^{d} \mathcal{D}(24)$, in the product (36), define cohomology classes for solutions of gravitational field equations in various types of gravity theories. For simplicity, we consider a four dimensional (4-d) nonholonomic manifold $\mathbf{V}$ with local coordinates $u^{\alpha}=\left(x^{1}, x^{2}, y^{3}=t, y^{4}\right)$ and signature $(++-+)$, i.e. $t$ is a time like coordinate. The Einstein equations with $\mathrm{N}$-anholonomic distributions on pseudoRiemannian and/or Lagrange-Finsler spaces with canonical d-connection/ normal $h-v$-connection / Cartan d-connection can be integrated in very general forms, see details and proofs in Refs. [34, 31. In this section, we show how general classes of exact solutions of Einstein equations can be generated as almost Kähler structures and classified following conditions of the algebraic index Theorem 4.2 ,

\subsubsection{Einstein-Finsler and Einstein spaces}

Einstein spaces, with the Ricci tensor proportional to the metric tensor via a nontrivial cosmological constant, and its polarizations can be constructed in various types of gravity theories. They are used for different 
studies of properties of gravitational vacuum and/or as the simplest approximations for matter, or extra dimension, contributions. Using $\mathrm{N}$-connection distributions and nonholonomic transforms, we can model Finsler configurations as exact solutions of gravitational field equations in general relativity and, inversely, a Finsler gravity model on tangent bundle can be constructed similarly to the Einstein gravity but for a different class of Finsler $\mathrm{d}-$-connections, see details and discussions in [35, 31.

\section{Proposition 4.2 -Definition.}

1. A nonholonomic Einstein space for the normal $d$-connection $\widehat{\mathbf{D}}(10)$ and $d$-metric $\mathbf{g}_{\alpha \beta}$ (17) is defined by solutions of Einstein equations (A.11) with source

$$
\begin{gathered}
\mathbf{\Upsilon}_{\delta}^{\alpha}=\operatorname{diag}\left[{ }^{v} \lambda\left(x^{k}, t\right),{ }^{v} \lambda\left(x^{k}, t\right) ;{ }^{h} \lambda\left(x^{k}\right),{ }^{h} \lambda\left(x^{k}\right)\right], \\
\widehat{\mathbf{R}}_{\beta \delta}=\mathbf{\Upsilon}^{\alpha}{ }_{\delta} .
\end{gathered}
$$

2. We construct geometric and physicial models of Einstein-Finsler/Lagrange spaces if $\widehat{\mathbf{D}}$ and $\mathbf{g}_{\alpha \beta}={ }^{F} \mathbf{g}_{\alpha \beta}$, or $={ }^{L} \mathbf{g}_{\alpha \beta}$, are taken for a Finsler/-Lagrange geometry. Almost Kähler models of Einstein/Finsler/-Lagrange spaces are elaborated in variables $\theta(\cdot, \cdot) \doteqdot \mathbf{g}(\mathbf{J} \cdot, \cdot)$ and $\widehat{\mathbf{D}} \equiv{ }_{\theta} \widehat{\mathbf{D}}$ for correspondingly prescribed $N$-connection structures.

3. As a particular case, we extract Einstein spaces for the Levi-Civita connection $\nabla$ if we impose additionally the conditions (A.12) and consider sources ${ }^{v} \lambda={ }^{h} \lambda=\lambda=$ const.

Proof. The first statement with equations (38) follows by contraction of indices in equations (A.11) with source (37). Such equations define "standard" Einstein-Finsler spaces if $\widehat{\mathbf{D}}$ and $\mathbf{g}_{\alpha \beta}$ are considered on $T M$.

The second statement can be derived for any frame/coordinate transforms

$$
\mathbf{f}_{\alpha \beta}=\mathbf{e}_{\alpha}^{\alpha^{\prime}} \mathbf{e}_{\beta}^{\beta^{\prime}} \mathbf{g}_{\alpha^{\prime} \beta^{\prime}},
$$

where $\mathbf{f}_{\alpha \beta}$ is a Finsler/ Lagrange metric of type (7), induced by a Hessian (2) , and $\mathbf{g}_{\alpha^{\prime} \beta^{\prime}}$ is a solution of (38). In $4-\mathrm{d}$ spaces, we have to find 16 coefficients $\mathbf{e}_{\alpha}^{\alpha^{\prime}}$, with given maximum 6 independent coefficients $\mathbf{g}_{\alpha^{\prime} \beta^{\prime}}$. By local coordinate transforms, and because of Bianchi identities, we can put zero 4 coefficients from 10 ones of a second rank symmetric tensor. In such way, we can also such way chose a generating Lagrange/Finsler function $L(x, y)$, or $\mathcal{L}(x, y)$, when the equations (39) can be solved on local cartes which should be neighborhoods, or charts throughout. Values $\mathbf{e}_{\alpha}^{\alpha^{\prime}}$ together with $\mathrm{N}$-connection coefficients $N_{i}^{a}$ induced by a corresponding $L / \mathcal{L}$ state the nonholonomic distribution which is considered for our Einstein-Finsler spacetime model. We 
conclude that a solution $\mathrm{g}$ of Einstein equations (38) can be written in Nadapted nonholonomic variables for some data $\left(g_{i j}, h_{a b}, N_{i}^{a}\right)$ encoding $\mathbf{g}_{\alpha \beta}$ in local coordinates, or (equivalently) in Finsler variables $\left(\mathbf{f}_{\alpha \beta},{ }^{c} N_{i}^{a}, \widehat{\mathbf{D}}\right)$, via frame transform (8) and (39). The priority of Finsler variables in various models of gravity is that choosing $\theta(\cdot, \cdot) \doteqdot \mathbf{g}(\mathbf{J} \cdot, \cdot)$ and $\widehat{\mathbf{D}} \equiv{ }_{\theta} \widehat{\mathbf{D}}$ we uniquely define compatible with $\theta,{ }_{\theta} \widehat{\mathbf{D}} \theta=0$, almost Kähler geometric models. This allows us to apply the Fedosov quantization methods.

The third statement is a consequence of the fact that the constraints $\widehat{L}_{a j}^{c}=e_{a}\left(N_{j}^{c}\right), \widehat{C}_{j b}^{i}=0, \Omega^{a}{ }_{j i}=0$ (A.12) are satisfied for the tensors $\widehat{\mathbf{T}}_{\alpha \beta}^{\gamma}(\mathrm{A.5})$ and then these $Z_{\alpha \beta}^{\gamma}(\mathrm{A.8})$ are zero. This states that $\widehat{\boldsymbol{\Gamma}}_{\alpha \beta}^{\gamma}=\Gamma_{\alpha \beta}^{\gamma}$, with respect to $\mathrm{N}$-adapted frames (5) and (6), see (A.9), even $\widehat{\mathbf{D}} \neq \nabla$.

\subsubsection{On "general" exact solutions in gravity}

Let us consider a metric parameterized with respect to a $\mathrm{N}$-adapted cobase (6) in the form

$$
\begin{aligned}
{ }^{\circ} \mathbf{g} & =e^{\psi\left(x^{k}\right)} d x^{i} \otimes d x^{i}+h_{3}\left(x^{k}, t\right) \mathbf{e}^{3} \otimes \mathbf{e}^{3}+\omega^{2}\left(x^{k}, t, x^{4}\right) h_{4}\left(x^{k}, t\right) \mathbf{e}^{4} \otimes \mathbf{e}^{4}, \\
\mathbf{e}^{3} & =d t+w_{i}\left(x^{k}, t\right) d x^{i}, \mathbf{e}^{4}=d y^{4}+n_{i}\left(x^{k}, t\right) d x^{i} .
\end{aligned}
$$

In brief, we denote $\partial a / \partial x^{1}=a^{\bullet}, \partial a / \partial x^{2}=a^{\prime}$ and $\partial a / \partial t=a^{*}$.

Proposition 4.3 For $h_{3,4}^{*} \neq 0$, the system of gravitational field equations defining Einstein-Finsler spaces for a normal $d$-connection and a metric (40) transform into

$$
\begin{aligned}
\ddot{\psi}+\psi^{\prime \prime} & =2{ }^{h} \lambda\left(x^{k}\right), \\
h_{4}^{*} & =2 h_{3} h_{4}{ }^{v} \lambda\left(x^{i}, t\right) / \phi^{*}, \\
\beta w_{i}+\alpha_{i} & =0 \\
n_{i}^{* *}+\gamma n_{i}^{*} & =0 \\
\mathbf{e}_{k} \omega=\partial_{4} \omega+w_{k} \omega^{*}+n_{k} \partial \omega / \partial y^{4} & =0
\end{aligned}
$$

where

$$
\phi=\ln \left|\frac{h_{4}^{*}}{\sqrt{\left|h_{3} h_{4}\right|}}\right|, \alpha_{i}=h_{4}^{*} \partial_{i} \phi, \beta=h_{4}^{*} \phi^{*}, \gamma=\left(\ln \left|h_{4}\right|^{3 / 2} /\left|h_{3}\right|\right)^{*} .
$$

Proof. For $\omega=1$, general proofs are contained in Refs. 31. In 34, there are provided respectively general solutions for nontrivial $\omega\left(x^{k}, t, x^{4}\right)$ and for the so-called Cartan connection in Finsler geometry. Such a proof consists of straightforward computations of the Ricci d-tensors for the dconnections under consideration, and equation (40). For some special cases when $h_{3}^{*}=0$, or $h_{4}^{*}=0$, and/or $\phi^{*}=0$, similar systems of equations can be derived. The rest of the proof is a lengthy and cumbersome computation that we leave to the reader. 
The above system of equations is with splitting of equations (not be confused with splitting of variables) which allows us to construct exact solutions in very general forms.

Theorem 4.3 If a metric $\mathbf{g}_{\alpha \beta}$ (17) in general relativity and/or EinsteinFinsler gravity can be related via nonholonomic transform to an ansatz (40), such a metric defines respectively an Einstein and/or Einstein-Finsler space.

Proof. We sketch the proof of $4-\mathrm{d}$ and conditions $h_{3,4}^{*} \neq 0$ (in [34, there are provided formulas for arbitrary dimensions for different classes of $\mathrm{d}$-connections). If $h_{4}^{*} \neq 0 ; \Upsilon_{2} \neq 0$, we get $\phi^{*} \neq 0$. Prescribing any nonconstant $\phi=\phi\left(x^{i}, t\right)$ as a generating function, we can construct exact solutions of (41)-(44): We solve step by step the two dimensional Laplace equation, for $g_{1}=g_{2}=e^{\psi\left(x^{k}\right)}$; integrate on $t$, in order to define $h_{3}, h_{4}$ and $n_{i}$; and finally solve the algebraic equations, for $w_{i}$. Finally, the solutions are obtained (computing consequently for a chosen $\phi\left(x^{k}, t\right)$ )

$$
\begin{aligned}
g_{1} & =g_{2}=e^{\psi\left(x^{k}\right)}, h_{3}= \pm \frac{\left|\phi^{*}\left(x^{i}, t\right)\right|}{{ }^{v} \lambda\left(x^{i}, t\right)} \\
h_{4} & ={ }^{0} h_{4}\left(x^{k}\right) \pm 2 \int \frac{\left(\exp \left[2 \phi\left(x^{k}, t\right)\right]\right)^{*}}{{ }^{v} \lambda\left(x^{i}, t\right)} d t \\
w_{i} & =-\partial_{i} \phi\left(x^{i}, t\right) / \phi^{*}\left(x^{i}, t\right), \\
n_{i} & ={ }^{1} n_{k}\left(x^{i}\right)+{ }^{2} n_{k}\left(x^{i}\right) \int\left[h_{3}\left(x^{i}, t\right) /\left(\sqrt{\left|h_{4}\left(x^{i}, t\right)\right|}\right)^{3}\right] d t,
\end{aligned}
$$

where ${ }^{0} h_{4}\left(x^{k}\right),{ }^{1} n_{k}\left(x^{i}\right)$ and ${ }^{2} n_{k}\left(x^{i}\right)$ are integration functions. In these formulas, we have to fix a corresponding sign \pm in order to generate a necessary local signature of type $(++-+)$ for some chosen $\phi, \Upsilon_{2}$ and $\Upsilon_{4}$. The function $\omega^{2}\left(x^{k}, t, x^{4}\right)$ can be an arbitrary one constrained to the condition (45). Such d-metrics generate Einstein-Finsler spaces for metric compatible Finsler d-connections.

To extract exact solutions in general relativity, i.e. for the Levi-Civita connection, we have to constrain the coefficients (47) of metric (40) to satisfy the conditions (A.12) and consider sources ${ }^{v} \lambda={ }^{h} \lambda=\lambda=$ const. This imposes additional constraints on the classes of generating and integration functions. We can select a subclass of Einstein spaces when ${ }^{2} n_{k}\left(x^{i}\right)=0$ and ${ }^{1} n_{k}\left(x^{i}\right)$ are subjected to conditions $\partial_{i}{ }^{1} n_{k}=\partial_{k}{ }^{1} n_{i}$. For $w_{i}=-\partial_{i} \phi / \phi^{*}$, we get functional constraints on $\phi\left(x^{k}, t\right)$, when

$$
\begin{array}{r}
\left(w_{i}[\phi]\right)^{*}+w_{i}[\phi]\left(h_{4}[\phi]\right)^{*}+\partial_{i} h_{4}[\phi]=0, \\
\partial_{i} w_{k}[\phi]=\partial_{k} w_{i}[\phi],
\end{array}
$$

where, for instance, we denoted by $h_{4}[\phi]$ the functional dependence on $\phi$.

Finally, we emphasize that the generic off-diagonal ansatz (40) define a very general class of exact solutions of gravitational field equations depending 
on all coordinates. Any metric related by frame transforms with a solution (47), $g_{\alpha \beta}=\mathbf{e}_{\alpha}^{\alpha^{\prime}} \mathbf{e}_{\beta}^{\beta^{\prime}}{ }^{\circ} \mathbf{g}_{\alpha^{\prime} \beta^{\prime}}$ also defines an exact solution. And inversely, for very general assumptions, if a metric $g_{\alpha \beta}$ is a solution of Einstein/Finsler equations, such a metric can be such a way parametrized by certain prescribed N-anholonomic distributions that an ansatz ${ }^{\circ} \mathbf{g}_{\alpha^{\prime} \beta^{\prime}}$ will be constructed (with corresponding generating and integration functions).

\subsubsection{Almost Kähler-Finsler variables}

In this subsection we show that for any given solution of Einstein equations, ${ }^{\circ} \mathbf{g}$ (40), on a nonholonomic manifold/bundle $\mathbf{V}, \operatorname{dim} \mathbf{V}=4$, we can introduce such a parametrization for the nonholonomic structure when the corresponding N-adapted geometric objects induce an almost Kähler structure. We put a left label " o" to values determining an exact solution for an Einstein and/or Einstein-Finsler space.

Finsler variables are introduced as solutions (on any chart or neighborhood) of algebraic equations $\mathbf{f}_{\alpha \beta}=\mathbf{e}_{\alpha}^{\alpha^{\prime}} \mathbf{e}^{\beta^{\prime}}{ }_{\beta}^{\circ} \mathbf{g}_{\alpha^{\prime} \beta^{\prime}}$ (39), where $\mathbf{f}_{\alpha \beta}$ is a Sasaki type d-metric (7) with coefficients generated by a regular $L=F^{2}$, or $\mathcal{L}=\mathcal{F}^{2}$, in the form $f_{a b}(2)$ and ${ }^{c} N_{i}^{a}$ (41) and ${ }^{\circ} \mathbf{g}_{\alpha^{\prime} \beta^{\prime}}$ is a general solution of type (40). We can fix the parameterizations, fix certain types of generating and integration functions, additional frame/coordinate transforms etc when some solutions of $\mathbf{e}_{\alpha}^{\alpha^{\prime}}$ are in a "simple" diagonal form. For instance, we can write in explicit $\mathrm{h}$ - and $\mathrm{v}$-components

$$
\begin{aligned}
& f_{i j}=e^{i^{\prime}}{ }_{i} e^{j^{\prime}}{ }^{\circ} g_{i^{\prime} j^{\prime}} \text { and } f_{a b}=e^{a^{\prime}}{ }_{a} e^{b^{\prime}}{ }_{b}^{\circ} g_{a^{\prime} b^{\prime}} \text {, } \\
& { }^{\circ} N_{i^{\prime}}^{a^{\prime}}=e_{i^{\prime}}{ }^{i} a_{a}^{a^{\prime}}{ }^{c} N_{i}^{a} \text {, or }{ }^{c} N_{i}^{a}=e_{i}^{i^{i^{\prime}}} e^{a}{ }_{a^{\prime}}{ }^{\circ} N_{i^{\prime}}^{a^{\prime}} \text {, }
\end{aligned}
$$

were, for instance, $e_{a^{\prime}}^{a}$ is inverse to $e_{a}^{a^{\prime}}$. Let us consider that ${ }^{\circ} g_{i^{\prime} j^{\prime}}=$ $\operatorname{diag}\left[{ }^{\circ} g_{1^{\prime}},{ }^{\circ} g_{2^{\prime}}\right], h_{a^{\prime} b^{\prime}}=\operatorname{diag}\left[{ }^{\circ} h_{3^{\prime}},{ }^{\circ} h_{4^{\prime}}\right]$ and ${ }^{\circ} N_{i^{\prime}}^{a^{\prime}}=\left\{{ }^{\circ} N_{i^{\prime}}^{3^{\prime}}=w_{i^{\prime}},{ }^{\circ} N_{i^{\prime}}^{4^{\prime}}=\right.$ $\left.n_{i^{\prime}}\right\}$ and (pseudo) Finsler data are $f_{i j}, f_{a b}$ and ${ }^{c} N_{i}^{a}=\left\{{ }^{c} N_{i}^{3}={ }^{c} w_{i},{ }^{c} N_{i}^{4}=\right.$ $\left.{ }^{c} n_{i}\right\}$, being parameterized by diagonal matrices, $f_{i j}=\operatorname{diag}\left[f_{1}, f_{2}\right]$ and $f_{a b}=\operatorname{diag}\left[f_{3}, f_{4}\right]$, if the generating function is of type $F={ }^{1} F\left(x^{i}, y^{3}\right)$ $+{ }^{2} F\left(x^{i}, y^{4}\right)$ for some homogeneous (respectively, on $y^{3}$ and $y^{4}$ ) functions ${ }^{1} F$ and ${ }^{2} F$. We may use arbitrary generating functions $F\left(x^{i}, y^{a}\right)$ but this will result in off-diagonal (pseudo) Finsler metrics in $\mathrm{N}$-adapted bases, which would request a more cumbersome matrix calculus.

For simplicity, we can fix such nonholonomic distributions (fixing correspondingly some generating/integration functions etc) when the conditions (49) are satisfied for a diagonal representation for $\mathbf{e}_{\alpha}^{\alpha^{\prime}}$,

$$
e_{1}^{1^{\prime}}= \pm \sqrt{\left|\frac{f_{1}}{{ }^{\circ} g_{1^{\prime}}}\right|}, e^{2^{\prime}}= \pm \sqrt{\left|\frac{f_{2}}{{ }^{\circ} g_{2^{\prime}}}\right|}, e_{3}^{3^{\prime}}= \pm \sqrt{\left|\frac{f_{3}}{{ }^{\circ} h_{3^{\prime}}}\right|}, e_{4}^{4^{\prime}}= \pm \sqrt{\left|\frac{f_{4}}{{ }^{\circ} h_{4^{\prime}}}\right|} .
$$

For any chosen values $f_{i}, f_{a}$ and ${ }^{c} w_{i},{ }^{c} n_{i}$ and given ${ }^{\circ} g_{i^{\prime}}$ and ${ }^{\circ} h_{a^{\prime}}$, we can 
compute ${ }^{\circ} w_{i^{\prime}}$ and ${ }^{\circ} n_{i^{\prime}}$ as

$$
\begin{aligned}
& { }^{\circ} w_{1^{\prime}}= \pm \sqrt{\left|\frac{{ }^{\circ} g_{1^{\prime}} f_{3}}{{ }^{\circ} h_{3^{\prime}} f_{1}}\right|}{ }^{c} w_{1}, \quad{ }^{\circ} w_{2^{\prime}}= \pm \sqrt{\left|\frac{{ }^{\circ} g_{2^{\prime}} f_{3}}{{ }^{\circ} h_{3^{\prime}} f_{2}}\right|} c^{c} w_{2}, \\
& { }^{\circ} n_{1^{\prime}}= \pm \sqrt{\left|\frac{{ }^{\circ} g_{1^{\prime}} f_{4}}{{ }^{\circ} h_{4^{\prime}} f_{1}}\right|}{ }^{c} n_{1}, \quad{ }^{\circ} n_{2^{\prime}}= \pm \sqrt{\left|\frac{{ }^{\circ} g_{2^{\prime}} f_{4}}{{ }^{\circ} h_{4^{\prime}} f_{2}}\right|}{ }^{c} n_{2},
\end{aligned}
$$

corresponding to solutions of equations (50).

Corollary 4.2 Any class of exact solutions of Einstein equations in general relativity and/or Einstein-Finsler gravity, depending on corresponding sets of generating/integration functions, defines a respective class of canonical almost Kähler structures.

Proof. It is a result of Theorems 2.2, 2.4 and 4.3, We have classes of equivalence for data (an explicit example is given by formulas (49) and (50))

$$
\begin{aligned}
\left(\mathbf{g}_{\alpha \beta}, N_{i}^{a}, \mathbf{D}_{\alpha}\right) & \sim\left({ }^{\circ} \mathbf{g}_{\alpha \beta},{ }^{\circ} N_{i}^{a},{ }^{\circ} \widehat{\mathbf{D}}_{\alpha}\right) \sim \\
\left(\mathbf{f}_{\alpha \beta},{ }^{c} N_{i}^{a},{ }^{c} \widehat{\mathbf{D}}_{\alpha}\right) & \sim\left({ }^{\circ} \theta(\cdot, \cdot) \doteqdot{ }^{\circ} \mathbf{g}(\mathbf{J} \cdot, \cdot), \quad{ }^{\circ} \mathbf{J},{ }^{c} \widehat{\mathbf{D}} \equiv{ }_{\theta} \widehat{\mathbf{D}}\right) .
\end{aligned}
$$

For Einstein manifolds, in general relativity, i.e. to encode data with the Levi-Civita connection $\nabla$, we have to consider additional constraints of type (A.12), when ${ }^{c} \widehat{\mathbf{D}} \rightarrow \nabla$.

The above mentioned classes of exact solutions expressed in various forms with nonholonomic/ Finsler / almost Kähler etc variables depend on the type of generating/integration functions we chose, and what type of, for instance, group/topological etc symmetries we prescribe for our geometric and/or physical models. In a series of our works, see reviews of results in [31, we constructed a number of examples with Finsler like, and non-Finsler, black ellipsoid, Taub NUT, solitonic, noncommutative, fractional etc gravitational solutions. It is an important task to elaborate certain criteria for algebraic classifications of such families of solutions; the geometric constructions should not depend explicitly on the type of generating/integration functions.

\subsubsection{Algebraic index classification of Einstein and Finsler spaces}

In addition to Petrov's algebraic classification of Riemannian and Weyl curvatures 27] and related gravitational field configurations in general relativity, we may provide a different algebraic classification of gravitational fields, using Atiyah-Singer theorem for nonholonomic almost Kähler manifolds. Such an index classification is related to Fedosov deformation quantization and can be performed for standard Einstein fields and modifications. 
Claim 4.1 Two solutions, $\left({ }_{1}^{\circ} \mathbf{g},{ }_{1}^{c} \widehat{\mathbf{D}}\right)$ and $\left({ }_{2}^{\circ} \mathbf{g},{ }_{2}^{c} \widehat{\mathbf{D}}\right)$, of Einstein equations (38) inducing two different nonholonomic almost Kähler structures, $\left({ }_{1}^{\circ} \theta,{ }_{\theta}^{1} \widehat{\mathbf{D}}\right)$ and $\left({ }_{2}^{\circ} \theta,{ }_{\theta}^{2} \widehat{\mathbf{D}}\right)$, i.e. two different ${ }_{1}^{\mathcal{L}} \mathbf{K}^{2 n}$ and ${ }_{2}^{\mathcal{L}} \mathbf{K}^{2 n}$, are noholonomically equivalent and characterized by the same model of Fedosov quantization if such solutions can be related via nonholonomic frame transforms, ${ }_{1}^{\circ} \mathbf{g}_{\alpha \beta}=$ $\mathbf{e}_{\alpha}^{\alpha^{\prime}} \mathbf{e}_{\beta}^{\beta_{2}^{\prime}} \stackrel{\circ}{2} \mathbf{g}_{\alpha^{\prime} \beta^{\prime}}$, and they have the same cohomology classes

$$
\operatorname{cl}\left({ }_{1}^{0} k\right)=\operatorname{cl}\left({ }_{2}^{0} k\right),
$$

for any elements ${ }_{1}^{0} k \in K_{0}\left({ }_{c}^{+} \mathcal{A}_{1}\right)$ and ${ }_{2}^{0} k \in K_{0}\left({ }_{c}^{+} \mathcal{A}_{2}\right)$ (respectively, for ${ }_{c}^{+} \mathcal{A}_{1}=C\left({ }_{1}^{\mathcal{L}} \mathbf{K}^{2 n}\right)[[v]]$ and $\left.{ }_{c}^{+} \mathcal{A}_{2}=C\left({ }_{2}^{\mathcal{L}} \mathbf{K}^{2 n}\right)[[v]]\right)$, when

$$
\begin{gathered}
c l\left({ }_{1}^{0} k\right)=\left[\widehat{A}\left({ }_{1}^{\mathcal{L}} \mathbf{K}^{2 n}\right) \exp \left(\frac{1}{v} \widehat{\mathcal{C}}\right) \operatorname{ch}\left(\mathcal{K}\left({ }_{1}^{0} k\right)\right)\right]_{n+n}, \\
\operatorname{cl}\left({ }_{2}^{0} k\right)=\left[\widehat{A}\left({ }_{2}^{\mathcal{L}} \mathbf{K}^{2 n}\right) \exp \left(\frac{2}{v}\right) \operatorname{ch}\left(\mathcal{K}\left({ }_{2}^{0} k\right)\right)\right]_{n+n}, \\
{ }_{1}^{W} \widehat{\mathcal{C}}=-{ }_{1} \theta+{ }_{1}^{v} \Omega \text { and }{ }_{2}^{W} \widehat{\mathcal{C}}=-{ }_{2} \theta+{ }_{2}^{v} \Omega .
\end{gathered}
$$

Let us provide two important motivations for such a claim. Different classes of exact solutions in classical gravity are defined by different generating/integration functions and associated nonholonomic structures. Under general frame/coordinate transforms, the parameterizations for fundamental geometric objects change substantially. Such generic nonlinear gravitational systems can be characterized topologically via corresponding elliptic operators and their cohomology classes. This is also an explicit application of the algebraic index theorem in quantum gravity which allows us to decide if two quantizations (in a generalized Fedosov sense) of some nonholonomic gravitational configurations possess the same cohomological characteristics, or not.

\section{A Einstein-Finsler Gravity in Almost Symplectic Variables}

Gravitational field equations in Einstein gravity on a (pseudo) Riemannian $\mathbf{V}$, and for Finsler gravity on $T M$, can be written equivalently in terms of the Levi-Civita connection $\nabla$, and using the almost symplectic connection ${ }_{\theta} \widehat{\mathbf{D}}$, both completely defined by the same fundamental geometric objects. We summarize necessary formulas from [29, 30, 31]. We use the term EinsteinFinsler gravity for two different classes of gravity theories: the first one is for the usual general relativity written equivalently in Finsler variables and the second one is for Finsler gravity models on tangent bundles enabled with metric compatible d-connections. 


\section{A.1 Torsion and curvature of normal d-connection}

Any d-connection $\mathbf{D}$ is characterized respectively by its torsion and curvature tensors,

$$
\begin{aligned}
\mathbf{T}(\mathbf{X}, \mathbf{Y}) & \doteqdot \mathbf{D}_{\mathbf{X}} \mathbf{Y}-\mathbf{D}_{\mathbf{Y}} \mathbf{X}-[\mathbf{X}, \mathbf{Y}] \\
\mathbf{R}(\mathbf{X}, \mathbf{Y}) \mathbf{Z} & \doteqdot \mathbf{D}_{\mathbf{X}} \mathbf{D}_{\mathbf{Y}} \mathbf{Z}-\mathbf{D}_{\mathbf{Y}} \mathbf{D}_{\mathbf{X}} \mathbf{Z}-\mathbf{D}_{[\mathbf{X}, \mathbf{Y}]} \mathbf{Z}
\end{aligned}
$$

where $[\mathbf{X}, \mathbf{Y}] \doteqdot \mathbf{X Y}-\mathbf{Y X}$, for any vectors $\mathbf{X}$ and $\mathbf{Y}$.

For the normal/almost symplectic d-connection $\widehat{\mathbf{D}}={ }_{\theta} \widehat{\mathbf{D}}=\left\{\widehat{\boldsymbol{\Gamma}}_{\beta \gamma}^{\alpha}\right\}$ (10), we can consider the 1 -form $\widehat{\boldsymbol{\Gamma}}_{j}^{i}=\widehat{L}_{j k}^{i} e^{k}+\widehat{C}_{j k}^{i} \mathbf{e}^{k}$, where $e^{k}=d x^{k}$ and $\mathbf{e}^{k}=$ $d y^{k}+N_{i}^{k} d x^{k}$, we can prove that the Cartan structure equations are satisfied,

$$
d e^{k}-e^{j} \wedge \widehat{\boldsymbol{\Gamma}}_{j}^{k}=-\widehat{\mathcal{T}}^{i}, d \mathbf{e}^{k}-\mathbf{e}^{j} \wedge \widehat{\boldsymbol{\Gamma}}_{j}^{k}=-{ }^{v} \widehat{\mathcal{T}}^{i},
$$

and

$$
d \widehat{\boldsymbol{\Gamma}}_{j}^{i}-\widehat{\boldsymbol{\Gamma}}_{j}^{h} \wedge \widehat{\boldsymbol{\Gamma}}_{h}^{i}=-\widehat{\mathcal{R}}_{j}^{i} .
$$

The torsion 2-form $\widehat{\mathcal{T}}^{\alpha}=\left(\widehat{\mathcal{T}}^{i},{ }^{v} \widehat{\mathcal{T}}^{i}\right)=\widehat{\mathbf{T}}^{\alpha}{ }_{\tau \beta} \mathbf{e}^{\tau} \wedge \mathbf{e}^{\beta}$ in (A.3) is computed:

$$
\widehat{\mathcal{T}}^{i}=\widehat{C}_{j k}^{i} e^{j} \wedge \mathbf{e}^{k},{ }^{v} \widehat{\mathcal{T}}^{i}=\frac{1}{2} \Omega_{k j}^{i} e^{k} \wedge e^{j}+\left(\frac{\partial N_{k}^{i}}{\partial y^{j}}-\widehat{L}_{k j}^{i}\right) e^{k} \wedge \mathbf{e}^{j} .
$$

i.e. the coefficients of torsion $\widehat{\mathbf{T}}_{\beta \gamma}^{\alpha}(\underline{\mathrm{A} .1})$ are

$$
\widehat{T}_{j k}^{i}=0, \widehat{T}_{j c}^{i}=\widehat{C}^{i}{ }_{j c}, \widehat{T}_{i j}^{a}=\Omega_{i j}^{a}, \widehat{T}_{i b}^{a}=e_{b} N_{i}^{a}-\widehat{L}_{b i}^{a}, \widehat{T}_{b c}^{a}=0 .
$$

It should be noted that $\widehat{\mathbf{T}}$ vanishes on $\mathrm{h}$ - and $\mathrm{v}$-subspaces, i.e. $\widehat{T}_{j k}^{i}=0$ and $\widehat{T}_{b c}^{a}=0$, and the nontrivial $\mathrm{h}-\mathrm{v}-$ components are induced nonholonomically and defined canonically by component $\mathbf{g}$ and $\mathcal{L}$.

The curvature 2-form from (A.4) of $\widehat{\boldsymbol{\Gamma}}_{\beta \gamma}^{\alpha}$ is computed

$$
\widehat{\mathcal{R}}_{\gamma}^{\tau}=\widehat{\mathbf{R}}_{\gamma \alpha \beta}^{\tau} \mathbf{e}^{\alpha} \wedge \mathbf{e}^{\beta}=\frac{1}{2} \widehat{R}^{i}{ }_{j k h} e^{k} \wedge e^{h}+\widehat{P}^{i}{ }_{j k a} e^{k} \wedge \mathbf{e}^{a}+\frac{1}{2} \widehat{S}^{i}{ }_{j c d} \mathbf{e}^{c} \wedge \mathbf{e}^{d},
$$

when the nontrivial N-adapted coefficients of curvature $\widehat{\mathbf{R}}_{\beta \gamma \tau}^{\alpha}(\underline{\mathrm{A} .2}$ are

$$
\begin{aligned}
\widehat{R}^{i}{ }_{h j k} & =\mathbf{e}_{k} \widehat{L}^{i}{ }_{h j}-\mathbf{e}_{j} \widehat{L}^{i}{ }_{h k}+\widehat{L}_{h j}^{m} \widehat{L}^{i}{ }_{m k}-\widehat{L}_{h k}^{m} \widehat{L}^{i}{ }_{m j}-\widehat{C}^{i}{ }_{h a} \Omega^{a}{ }_{k j}, \\
\widehat{P}^{i}{ }_{j k a} & =e_{a} \widehat{L}^{i}{ }_{j k}-\widehat{\mathbf{D}}_{k} \widehat{C}^{i}{ }_{j a}, \widehat{S}^{a}{ }_{b c d}=e_{d} \widehat{C}^{a}{ }_{b c}-e_{c} \widehat{C}^{a}{ }_{b d}+\widehat{C}^{e}{ }_{b c} \widehat{C}^{a}{ }_{e d}-\widehat{C}^{e}{ }_{b d} \widehat{C}^{a}{ }_{e c}
\end{aligned}
$$

The N-adapted coefficients of the normal d-connection $\widehat{\mathbf{D}}={ }_{\theta} \widehat{\mathbf{D}}=$ $\left\{\widehat{\boldsymbol{\Gamma}}_{\beta \gamma}^{\alpha}\right\}$ and of the the Levi-Civita connection $\nabla=\left\{{ }_{1} \Gamma_{\alpha \beta}^{\gamma}\right\}$ are related via formulas

$$
{ }_{1} \Gamma_{\alpha \beta}^{\gamma}=\widehat{\boldsymbol{\Gamma}}_{\alpha \beta}^{\gamma}+{ }_{1} Z_{\alpha \beta}^{\gamma},
$$


where the distortion d-tensor,$Z_{\alpha \beta}^{\gamma}$ is computed

$$
\begin{aligned}
& Z_{j k}^{a}=-\widehat{C}_{j b}^{i} g_{i k} g^{a b}-\frac{1}{2} \Omega_{j k}^{a},, Z_{b k}^{i}=\frac{1}{2} \Omega_{j k}^{c} g_{c b} g^{j i}-\Xi_{j k}^{i h} \widehat{C}_{h b}^{j}, \\
& Z_{j k}^{i}=0, Z_{b k}^{a}={ }^{+} \Xi_{c d}^{a b} \quad \widehat{T}_{b k}^{c},, Z_{k b}^{i}=\frac{1}{2} \Omega_{j k}^{a} g_{c b} g^{j i}+\Xi_{j k}^{i h} \widehat{C}_{h b}^{j}, \\
& Z_{j b}^{a}=-{ }^{-} \Xi_{c b}^{a d} \widehat{T}_{d j}^{c}, Z_{b c}^{a}=0, Z_{a b}^{i}=-\frac{g^{i j}}{2}\left[\widehat{T}_{a j}^{c} g_{c b}+\widehat{T}_{b j}^{c} g_{c a}\right],
\end{aligned}
$$

for $e_{b}=\partial / \partial y^{a}$ and $\Xi_{j k}^{i h}=\frac{1}{2}\left(\delta_{j}^{i} \delta_{k}^{h}-g_{j k} g^{i h}\right),{ }^{ \pm} \Xi_{c d}^{a b}=\frac{1}{2}\left(\delta_{c}^{a} \delta_{d}^{b} \pm g_{c d} g^{a b}\right)$. The values (A.8) and (A.9), and the $\mathrm{h}-$ and $\mathrm{v}$-components of $\widehat{\boldsymbol{\Gamma}}_{\beta \gamma}^{\alpha}$ given by (10) are determined by coefficients of metric $\mathbf{g}$ on $\mathbf{V}$, and $\mathbf{N}$, for a prescribed nonholonomic distribution with associated $\mathrm{N}$-connection structure.

\section{A.2 The Einstein equations for almost symplectic $d-$ connec- tions}

The Ricci tensor $\widehat{R} i c=\left\{\widehat{\mathbf{R}}_{\alpha \beta}\right\}$ of $\widehat{\mathbf{D}}={ }_{\theta} \widehat{\mathbf{D}}$ can be defined in standard form by contracting respectively the components of (A.7), $\widehat{\mathbf{R}}_{\alpha \beta} \doteqdot \widehat{\mathbf{R}}_{\alpha \beta \tau}^{\tau}$. The scalar curvature is

$$
{ }^{s} \widehat{\mathbf{R}} \doteqdot \mathbf{g}^{\alpha \beta} \widehat{\mathbf{R}}_{\alpha \beta}=g^{i j} \widehat{R}_{i j}+h^{a b} \widehat{R}_{a b},
$$

where $\widehat{R}=g^{i j} \widehat{R}_{i j}$ and $\widehat{S}=h^{a b} \widehat{R}_{a b}$ are respectively the $\mathrm{h}-$ and v-components of scalar curvature. This allows a geometric formulation of the gravitational field equations for the almost symplectic connection,

$$
\widehat{\mathbf{E}}_{\beta \delta}=\widehat{\mathbf{R}}_{\beta \delta}-\frac{1}{2} \mathbf{g}_{\beta \delta}{ }^{s} R=\widehat{\boldsymbol{\Upsilon}}_{\beta \delta}
$$

We can state well defined conditions when (A.11) can be constructed to be equivalent to the Einstein equations for $\nabla$. This is possible if $\widehat{\boldsymbol{\Upsilon}}_{\beta \delta}=$ ${ }^{m} \mathbf{\Upsilon}_{\beta \delta}+{ }^{z} \mathbf{\Upsilon}_{\beta \delta}$ are derived in such a way that they contain contributions from 1) the N-adapted energy-momentum tensor ${ }^{m} \boldsymbol{\Upsilon}_{\beta \delta}$ (defined variationally following the same principles as in general relativity but on $\mathbf{V}$ ) and 2), the distortion of the Einstein tensor in terms of $\widehat{\mathbf{Z}}\left(\underline{\mathrm{A} .9)}, \widehat{\mathbf{E}}_{\beta \delta}={ }_{1} E_{\alpha \beta}+{ }^{z} \widehat{\mathbf{E}}_{\beta \delta}\right.$, for ${ }^{z} \widehat{\mathbf{E}}_{\beta \delta}={ }^{z} \boldsymbol{\Upsilon}_{\beta \delta} 1$

The equations (A.11) are considered as the fundamental field equations in Einstein-Finsler gravity (the d-connection ${ }_{\theta} \widehat{\mathbf{D}}$ is also a Finsler/Lagrange connection), see details in Refs. [31. They transform into usual Einstein equations in general relativity if

$$
\widehat{L}_{a j}^{c}=e_{a}\left(N_{j}^{c}\right), \widehat{C}_{j b}^{i}=0, \Omega^{a}{ }_{j i}=0,
$$

for $\Upsilon_{\beta \delta} \rightarrow \varkappa T_{\beta \delta}$ (matter energy-momentum in Einstein gravity) if $\widehat{\mathbf{D}} \rightarrow \nabla$.

\footnotetext{
${ }^{1}$ The value ${ }^{z} \widehat{\mathbf{E}}_{\beta \delta}$ is computed by introducing $\widehat{\mathbf{D}}=\nabla-\widehat{\mathbf{Z}}$ into A.11 and corresponding contractions of indices in order to find the Ricci d-tensor and scalar curvature.
} 


\section{B Proof of Main Theorem}

\section{B.1 A technical Lemma}

Let us prove an obvious "nonholonomic" analogue of Lemma 1 in 12 which have technical importance for proving the Main Result of this paper.

Lemma B.1 Let us consider a nonholonomic vector bundle $\mathcal{E}$ of rank $k$ over ${ }^{L} \mathbf{K}^{2 n}$ endowed with a Fedosov-Finsler normal d-connection ${ }_{r}^{d} \mathcal{D}=$ ${ }^{d} \mathcal{D}+v^{-1}\left[{ }^{\theta} r, \cdot\right]$ (24) when $\delta^{-1}{ }^{\theta} r=0$, following the conditions of Corollary 3.1. For any endomorphism of $\mathcal{E}, \varsigma \in E n d_{V}$, and connection ${ }^{\mathcal{E}} \partial$, if $\mathcal{E}_{\partial \varsigma}=0$ we get ${ }_{r}^{d} \mathcal{D} \varsigma=0$, i.e. the isomorphism $\chi$ (32) transforms $\varsigma$ into itself.

Proof. If $\varsigma$ does not depend on $z$-variables, i.e. ${ }^{\mathcal{E}} \partial \varsigma=0$, we have ${ }^{d} \mathcal{D} \varsigma=\delta \varsigma=0$ and ${ }_{r}^{d} \mathcal{D} \varsigma=v^{-1}\left[{ }^{\theta} r, \varsigma\right]$ when (for a nilpotent ${ }_{r}^{d} \mathcal{D}$ ) we must have ${ }_{r}^{d} \mathcal{D}\left[{ }^{\theta} r, \varsigma\right]=0$. For our purposes, we should prove that $\left[{ }^{\theta} r, \varsigma\right]$ vanishes.

Since $\delta^{-1}{ }^{\theta} r=0$ and $\varsigma$ does not depend on $z^{-}$-variables, $\delta^{-1}\left[{ }^{\theta} r, \varsigma\right]=0$. We compute $\delta\left[{ }^{\theta} r, \varsigma\right]={ }^{d} \mathcal{D}\left[{ }^{\theta} r, \varsigma\right]+v^{-1}\left[{ }^{\theta} r,\left[{ }^{\theta} r, \varsigma\right]\right]$, when $\left[{ }^{\theta} r, \varsigma\right] \in$ ${ }^{d} \Omega^{1}\left(\mathbf{W}\left(E n d_{V}\right)\right)$. Applying (29) to $\left[{ }^{\theta} r, \varsigma\right]$ we get the equation $\left[{ }^{\theta} r, \varsigma\right]=$ $\delta^{-1}\left({ }^{d} \mathcal{D}\left[{ }^{\theta} r, \varsigma\right]+v^{-1}\left[{ }^{\theta} r, \varsigma\right]\right)$. There is only one non-contradictory solution $\left[{ }^{\theta} r, \varsigma\right]=0$ because $\delta^{-1}$ increases the degree in $z$-variables, but such a commutator does not.

We emphasize that on ${ }^{\mathcal{L}} \mathbf{K}^{2 n}$ endowed with certain canonical symplectic forms and N-adapted connections we can work similarly as on Kähler manifolds but keeping the constructions to be distinguished nonholonomically as some $\mathrm{h}-$ and $\mathrm{v}-$-components of a corresponding almost Kähler geometry.

\section{B.2 Sketch of proof for theorem 4.2}

The method we should apply is inspired from [12], see there the Theorem 4. The idea is to prove that distinguished $(n+n)$-form ${ }^{\mathcal{D}} \digamma\left({ }^{p} \zeta_{1}\right)$ has the same cohomology class as the $(n+n)$-th component of the form

$$
\operatorname{det}\left(\frac{{ }^{s} \widehat{\mathbf{R}} / 2}{\sinh \left({ }^{s} \widehat{\mathbf{R}} / 2\right)}\right)^{1 / 2} e^{v^{-1}\left(\theta-{ }^{v} \Omega\right)} \operatorname{tr} e^{{ }^{V} \mathcal{R}},
$$

where ${ }^{s} \widehat{\mathbf{R}}$ A.10) is the scalar curvature of the normal d-connection, ${ }^{W} \widehat{\mathcal{C}}=$ $-\theta+{ }^{v} \Omega$, see (31), ${ }^{V} \mathcal{R}$ is the scalar curvature form on the d-vector bundle $\mathcal{E}$ and $t r$ is the ordinary trace of matrices. Such a formula is similar to that derived in Theorem 3.1 .

The above Lemma is necessary for evaluating principal parts and idempotents of involved $\mathrm{d}$-algebras. Computations are performed similarly for the $\mathrm{h}$ - and $\mathrm{v}$-components dubbing the formulas provided in the holonomic Kähler constructions. We leave the technical details to the interested reader. 


\section{References}

[1] a pair $(\mathbf{V}, \mathcal{N})$ consisting of a manifold $\mathbf{V}$ and a non-integrable distribution $\mathcal{N}$ on this space is called a nonholonomic manifold

[2] For different purposes, we can consider such a space to be a semiRiemannian/ Riemann-Cartan manifold, a real/ complex (co) vector and tangent bundle, a Clifford module; we can extend the approach to various noncommutative and supersymmetric generalizations etc.

[3] physicists use instead of "semi" the term "pseudo", for local pseudoEuclidean signatures of type $(-,+,+,+)$; in this paper, we consider that a pseudo-Riemannian spaces is a manifold of necessary smooth class and endowed with a metric structure of local pseudo-Euclidean signature

[4] we shall use ":=" instead of "by definition"; here we also note that a symbol like $\mathbf{e}_{i}$ is boldface because it contains N-elongated terms; $e_{b}$ is not boldface because it is just a partial derivative and do not include $\mathrm{N}$-coefficients; for dual values, we write $e^{i}$ but $\mathbf{e}^{b}$ because only in the second case there are $\mathrm{N}$-elongations

[5] M. Anastasiei and S. Vacaru, Fedosov quantization of Lagrange-Finsler and Hamilton-Cartan spaces and Einstein gravity lifts on (co) tangent bundles, J. Math. Phys. 50 (2009) 013510

[6] M. Atiyah and I. Singer, The index of elliptic operators, I, III, Ann. Math. 87 (1968) 484-530; 546-609

[7] A. Bejancu and H. R. Farran, Foliations and Geometric Structures (Springer 2005)

[8] P. Bressler, R. Nest and B. Tsygan, Riemann-Roch theorems via deformation quantization. I \& II, Adv.Math. 167 (2002) 1-25; 26-73

[9] L. Boutet de Monvel and V. Guillemin, The spectral theory of Toeplitz operators, Ann. Math. Stud., Vol. 99 (Princeton University Press, 1981)

[10] D. Calaque and M. Van den Bergh, Hochschild cohomology and Atiyah classes, in press: Adv. Math. (2010) arXiv: 0708.2725

[11] C. Castro, W-geometry from Fedosov's deformation quantization, J. Geom. Phys. 33 (2000) 173-190

[12] PoNing Chen and V. Dolgushev, A simple algebraic proof of the algebraic index theorem, Math. Research Letters 12 (2005) 655-672

[13] F. Etayo, R. Santamaría and S. Vacaru, Lagrange-Fedosov nonholonomic manifolds, J. Math. Phys. 46 (2005) 032901 
[14] B. Fedosov, A simple geometrical construction of deformation quantization, J. Diff. Geom. 40 (1994) 213-238

[15] B. Fedosov, Deformation Quantization and Index Theory, Mathematical topics: 9 (Akademie-Verlag, Berlin, 1996)

[16] B. Feigin, G. Felder and B. Shoikhet, Hochschild cohomology of the Weyl algebra and trace in deformation quantization, Duke Math. J. 127 (2005) 487-517

[17] I. Gelfand, V. Retakh and M. Shubin, Fedosov manifolds, Advances in Mathematics, 136 (1998) 104-140

[18] M. Grigoriev and S. Lyakhovich, Fedosov deformation quantization as a BRST theory, Commun. Math. Phys. 218 (2001) 437-457

[19] A. Karabegov, On the dequantization of Fedosov's deformation quantization, Lett. Math. Phys. 65 (2003) 133-146

[20] A. Karabegov, Fedosov's formal symplectic groupoids and contravariant connections, Commun. Math. Phys. 258 (2005) 223-356

[21] A. Karabegov and M. Schlichenmaier, Almost Kähler deformation quantization, Lett. Math. Phys. 57 (2001) 135-148

[22] J. Kern, Lagrange geometry, Arch. Math. 25 (1974) 438-443

[23] M. Kontsevich, Deformation quantization of Poisson manifolds, Lett. Math. Phys. 66 (2003) 157-216

[24] M. Kontsevich, Operads and motives in deformation quantization, Lett. Math. Phys. 48 (1999) 35-72

[25] M. Matsumoto, Foundations of Finsler Geometry and Special Finsler Spaces (Kaisisha: Shingaken, Japan, 1986)

[26] R. Nest and B. Tsygan, Algebraic index theory, Commun. Math. Phys. 172 (1995) 223-262

[27] A. Z. Petrov, Classification of spaces defined by gravitational fields. Uch. Zapiski Kazan Gos. Univ. 144 (1954) 55-75; English translation: Gen. Rel. Grav. 22 (2000) 1665-1685

[28] S. Vacaru, Deformation quantization of almost Kähler models and Lagrange-Finsler spaces, J. Math. Phys. 48 (2007) 123509

[29] S. Vacaru, Deformation quantization of nonholonomic almost Kähler models and Einstein gravity, Phys. Lett. A 372 (2008) 2949-2955 
[30] S. Vacaru, Einstein gravity as a nonholonomic almost Kähler geometry, Lagrange-Finsler variables, and deformation quantization; J. Geom. Phys. 60 (2010) 1289-1305

[31] S. Vacaru, Finsler and Lagrange geometries in Einstein and string gravity, Int. J. Geom. Methods. Mod. Phys. 5 (2008) 473-511

[32] S. Vacaru, Branes and quantization for an A-model complexification of Einstein gravity in almost Kähler variables, Int. J. Geom. Methods. Mod. Phys. 6 (2009) 873-909

[33] S. Vacaru, Nonholomomic distributions and gauge models of Einstein gravity, Int. J. Geom. Methods. Mod. Phys. 7 (2010) 215-246

[34] S. Vacaru, On general solutions in Einstein and higher dimension gravity, Int. J. Theor. Phys. 49 (2010) 884-913

[35] S. Vacaru, Critical remarks on Finsler modifications of gravity and cosmology by Zhe Chang and Xin Li; Phys. Lett. B (2010) 224-228

[36] S. Vacaru and J. F. Gonzalez-Hernandez, Nonlinear connections on gerbes, Clifford-Finsler modules, and the index theorems, Indian J. Math. 50 (2008) 573-606

[37] I. Vaisman, Symplectic curvature tenors, Monatshefte für Mathematik, 100 (1985) 299-327

[38] G. Vrănceanu, Sur les espaces non holonomes. C. R. Acad. Paris, 103 (1926) 852-854

[39] G. Vrănceanu, Sur quelques points de la théories des espaces non holonomomes, Bull. Fac. Şt. Cernăuţi 5 (1931) 177-205

[40] G. Vrănceanu, Leçons de Geometrie Differentielle, Vol. II (Edition de l'Academie de la Republique Populaire de Roumanie, 1957) 